\title{
The case for a rational genome-based vaccine against malaria
}

\section{Carla Proietti and Denise L. Doolan*}

Infectious Diseases Program, QIMR Berghofer Medical Research Institute, Brisbane, QLD, Australia

\section{Edited by:}

Thomas Laurence Richie, Thomas L. Richie, Consulting, USA

\section{Reviewed by:}

Xun Suo, China Agricultural University, China

Hajime Hisaeda, Gunma University, Japan

\section{${ }^{*}$ Correspondence:}

Denise L. Doolan, Infectious Diseases Program, QIMR Berghofer Medical Research Institute, Locked Bag 2000 Royal Brisbane Hospital, Brisbane, QLD 4029, Australia e-mail:Denise.Doolan@ qimrberghofer.edu.au
Historically, vaccines have been designed to mimic the immunity induced by natural exposure to the target pathogen, but this approach has not been effective for any parasitic pathogen of humans or complex pathogens that cause chronic disease in humans, such as Plasmodium. Despite intense efforts by many laboratories around the world on different aspects of Plasmodium spp. molecular and cell biology, epidemiology and immunology, progress towards the goal of an effective malaria vaccine has been disappointing. The premise of rational vaccine design is to induce the desired immune response against the key pathogen antigens or epitopes targeted by protective immune responses. We advocate that development of an optimally efficacious malaria vaccine will need to improve on nature, and that this can be accomplished by rational vaccine design facilitated by mining genomic, proteomic and transcriptomic datasets in the context of relevant biological function. In our opinion, modern genome-based rational vaccine design offers enormous potential above and beyond that of whole-organism vaccines approaches established over 200 years ago where immunity is likely suboptimal due to the many genetic and immunological hostparasite adaptations evolved to allow the Plasmodium parasite to coexist in the human host, and which are associated with logistic and regulatory hurdles for production and delivery.

Keywords: malaria, vaccine, rational vaccine design, antigen discovery, genome-based, protective immunity

\section{INTRODUCTION}

Vaccines are the most efficient health care intervention for preventing morbidity and mortality and improving public health, except for water sanitation (World Health Organization [WHO], 2014a). The field of vaccinology originated in 1796 when Edward Jenner protected James Phipps against smallpox by inoculation with cowpox (Jenner, 1798; Baxby, 1999; Tuells, 2012). However, despite dedicated efforts to develop vaccines against a range of viral, bacterial, or parasitic diseases, approximately one third of all deaths (at least 15 million people each year) and 68\% of deaths in children under 5 years of age $(5$ million children each year) are due to infectious diseases (World Health Organization [WHO], 2014b). Of these, three pathogens that cause chronic infections - the Plasmodium parasite, human immunodeficiency virus (HIV) and Mycobacterium tuberculosis (TB); known by public health officials as the "big three" - are the major threats responsible for $10 \%$ of all deaths globally and more than half the global burden of infectious diseases. Moreover, although there is an extensive vaccine portfolio against viral and bacterial pathogens, there are no licensed vaccines for any parasitic infection of humans or for any chronic infections by complex pathogens (World Health Organization [WHO], 2006; Moorthy and Kieny, 2010). Indeed, there is only one therapeutic vaccine approved by the US Food and Drug Administration (FDA) or European Medicines Agency (EMA), for a metastatic hormonerefractory prostate cancer (Provenge ${ }^{\circledR}$, DendreonCorp, USA) but this requires preparation of a personalized vaccine for each patient and so is expensive $(\sim \$$ US93,000) and has very poor uptake. New approaches for the development of vaccines against complex and chronic pathogens are urgently needed. Malaria is an excellent model for such approaches, being a complex pathogen which causes chronic infections and one of the "big three" public health targets.

The Malaria Vaccine Technology Road Map was published in 2006 as the result of a collective effort by the malaria vaccine community ${ }^{1}$. A comprehensive update to this roadmap was released in 2013 with the strategic goal to, by 2030, license vaccines targeting Plasmodium falciparum and $P$. vivax with a protective efficacy of at least $75 \%$ against clinical malaria with a duration of protection of at least 2 years and booster doses to be required no more frequently than annually ${ }^{2}$. In the intervening years, there has been a call for global malaria eradication, issued by Bill and Melinda Gates in October 2007 (Roberts and Enserink, 2007) and taken up the malaria community with a consensus community-based Malaria Eradication Agenda $^{3}$. Many experts consider that vaccines will play a key role in the eradication process (Hall and Fauci, 2009; Plowe et al., 2009) and vaccines will certainly be important to sustain and improve on levels of control achieved by other interventions such anti-malarial drugs, insecticide spraying and insecticideimpregnated bed nets. Additionally, the Global Vaccine Action Plan 2011-2020 compiled by stakeholders from the global health

\footnotetext{
${ }^{1}$ http://www.malariavaccine.org/files/Malaria_Vaccine_TRM_Final_000.pdf

${ }^{2}$ http://www.who.int/immunization/topics/malaria/vaccine_roadmap/en/

${ }^{3}$ http://www.who.int/malaria/elimination/maleraupdate.pdf
} 
community including the World Health Organization (WHO), the United Nations Children's Fund (UNICEF), the National Institute of Allergy and Infectious Diseases (NIAID), and the Bill \& Melinda Gates Foundation (BMGF) included as a key indicator of progress towards one of its six strategic objectives, "proof of concept for a vaccine that shows greater or equal to $75 \%$ efficacy for HIV/AIDS, tuberculosis or malaria” by 2020 (World Health Organization [WHO], 2012).

However, despite intense effort for many decades by researchers throughout the world, the development of a malaria vaccine that meets the stated goals remains elusive (Sherman, 2009). Almost all efforts have focused on the pre-erythrocytic or asexual blood stages of $P$. falciparum although recent efforts have been also directed to the sexual stage to provide 'herd immunity' rather than confer individual protection as a consequence of the malaria eradication agenda, as well as P. vivax (Schwartz etal., 2012; World Health Organization [WHO], 2014b). An efficacious pre-erythrocytic vaccine is considered ideal since it would halt the development of the parasite in the liver stage and thereby prevent the blood stage of the life cycle which is the stage associated with the development of clinical symptoms, as well as the transmission of the disease which occurs in the sexual stage. The most advanced malaria vaccine candidate is RTS,S, a hybrid virus-like particle containing the C-terminus of the $P$. falciparum circumsporozoite protein (CSP) fused to hepatitis B surface antigen, expressed in Saccharomyces cerevisiae together with hepatitis B surface antigen (Ballou and Cahill, 2007). Recently, regulatory approval for RTS,S/ASO1 targeted at infants aged 6-14 weeks and administered through the routine Expanded Program on Immunization (EPI) has been submitted to the European Medicines Agency by GlaxoSmithKline following pivotal Phase III evaluation at 11 sites in sub-Saharan Africa; if the required regulatory approvals are obtained and the Phase III safety and efficacy data are satisfactory, the WHO has indicated that a policy recommendation for RTS,S may be granted in $2015^{4}$. Although modeling estimates predict that this vaccine should have a public health impact in terms of number of "deaths averted” (Brooks et al., 2012; Nunes et al., 2013), the Phase III efficacy of the vaccine for its intended outcome in the target age group is very low and is not sustained (Duncan and Hill, 2011; Olotu et al., 2013). Specifically, after 18 months of followup, efficacy against clinical malaria was only $27 \%$ among infants aged 6-12 weeks at the first vaccination; among children aged 517 months at first vaccination, vaccine efficacy was 46\%; moreover, these results were achieved on top of existing malaria interventions including insecticide-treated bed nets used by 86 and 78\% of trial participants aged 6-12 weeks or 5-17 months, respectively $^{5}$. Although the RTS,S data are encouraging, this milestone has taken almost 30 years of extensive preclinical and clinical development by GSK in partnership with the US Army, supported by more than US\$200 million from the Bill \& Melinda Gates Foundation in addition to more than \$350 GSK funds to date, with an additional $\$ 260$ million investment anticipated ${ }^{5}$ (Ballou and Cahill, 2007).

\footnotetext{
${ }^{4} \mathrm{http}: / /$ www.malariavaccine.org/files/MVI-GSK-RTSSfactsheetFINAL-web.pdf

${ }^{5} \mathrm{http}: / /$ www.malariavaccine.org/files/MVI-GSK-FAQ-FINAL-web.pdf
}

\section{CHALLENGES FOR MALARIA VACCINE DEVELOPMENT}

The development of an effective vaccine against malaria has been hindered by the complexity of the Plasmodium spp. parasite as well as the host response to the parasite. Recent evidence indicates that the Plasmodium parasite has co-evolved with the human host for 1000s of years (Liu et al., 2010) with evolutionary coadaptation allowing for chronic persistence of the parasite in the human host, and recurrent infections (Pierce and Miller, 2009). The Plasmodium life cycle involves both invertebrate (mosquito) and vertebrate (mammalian) hosts, with multiple stages within the host (sporozoite, liver, asexual blood stage, sexual) and numerous intracellular and extracellular environments in which the parasite develops (Langhorne et al., 2008). Different host responses are required to target these distinct life cycle stages - primarily antibodies against the extracellular parasite stages exposed in the peripheral circulation (sporozoites, asexual blood stage merozoites) and $\mathrm{T}$ cells against the intracellular stages (hepatic/liver stages) which are not accessible to circulating antibodies. In addition, the Plasmodium spp. parasite has a large 23 megabase genome that contains an estimated 5,300 putative proteins, many of which are expressed in different stages of the life cycle (Gardner et al., 2002). Moreover, many of these protein may exhibit allelic polymorphism (more than one allele existing for certain regions of a protein, e.g., MSP1 or AMA1), antigenic polymorphism (point mutations at the nucleotide level, e.g., CSP) often localized to B cell or T cell epitopes, or antigenic variation (multi-copy variant surfaces antigens, e.g., var genes, rifins; Good et al., 1988; Takala and Plowe, 2009; Kirkman and Deitsch, 2012; Barry and Arnott, 2014). A vaccine must be effective against all antigenic or allelic variants responses, to ensure efficacy against all variant circulating strains in the field (Moorthy and Kieny, 2010). In addition to this selection by the parasite of mutations that avoid the host protective immune response and confer susceptibility to the disease, there is selection by humans of mutations that confer resistance. Indeed, malaria is considered to have had the greatest impact of all pathogens in shaping the human genome with evidence that a number of genetic polymorphisms within the human genome, including $\alpha$ thalassemia and hemoglobinopathies such as the sickle-cell trait, have arisen due to evolutionary pressure exerted by the Plasmodium parasite (Mackinnon and Marsh, 2010; Taylor et al., 2013). The high mortality rate associated with malaria would be predicted to exert a powerful selective pressure on the human genome by positively selecting any genetic mutation that confers protection against death to allow the Plasmodium parasite to persist in the host and be transmitted (Pierce and Miller, 2009; Mackinnon and Marsh, 2010). Other sophisticated immune evasion strategies at the host-parasite interface include the ability of the parasite to modulate the host immune response, reviewed extensively elsewhere (Langhorne et al., 2008; Casares and Richie, 2009; Pierce and Miller, 2009). These complex host-parasite interactions at the genetic and immunological levels pose significant challenges for vaccine development.

\section{FEASIBILITY OF VACCINATION AGAINST MALARIA}

In spite of these challenges, there is growing evidence supporting the feasibility of developing an effective malaria vaccine. Field studies have demonstrated a decreasing incidence and density 
of infection with age and exposure to natural infection, and reduced frequency and severity of clinical illness, indicative of the acquisition of anti-disease immunity (Baird, 1998; Doolan et al., 2009). Also, passive transfer of polyclonal sera or purified immunoglobulin from individuals with lifelong exposure to $P$. falciparum resulted in a significant reduction in bloodstage parasitemia and recovery from clinical symptoms (Cohen et al., 1961; Sabchareon et al., 1991). Those studies implicate antibodies directed against blood stage antigens as the key immune effectors in naturally acquired immunity. This protection is antidisease immunity but not anti-parasite immunity since most individuals with long-term exposure in malaria endemic areas who have developed effective clinical immunity will nonetheless continue to experience low-density, asymptomatic infections (Okell et al., 2009). Longitudinal studies in malaria-endemic populations suggest that immune responses to the pre-erythrocytic stages probably have limited involvement in this anti-disease immunity and that immunity to the pre-erythrocytic stage is not naturally acquired (Owusu-Agyei et al., 2001; Tran et al., 2013). Nonetheless, epidemiological studies suggest that exposure to low numbers of sporozoites, although not sterilizing, can reduce the parasite load in the liver and lower blood stage parasitemia, since the intensity of exposure to biting infectious mosquitoes (entomological inoculation rate) has been significantly associated with the incidence and density (but not prevalence) of $P$. falciparum parasitemia in children (Doolan et al., 2009).

More convincing evidence for the feasibility of vaccination against malaria exists from studies focused on the experimental induction of protective immunity, which have established that sterile infection-blocking protective immunity directed against the pre-erythrocytic stage can be achieved in mice and humans. Considered for many years the "gold standard" for malaria vaccine development, sterile protection can be induced in mice, non-human primates and humans by exposure to the bites of radiation-attenuated $P$. yoelii, $P$. berghei, $P$. knowlesi, or $P$. falciparum infected mosquitoes, or by intravenous immunization with isolated irradiated sporozoites, provided that the dose of radiation is sufficient to attenuate the parasite such that it can invade the hepatocyte but not develop into the blood-stage (Nussenzweig et al., 1967; Gwadz et al., 1979; Hoffman et al., 2002; Weiss and Jiang, 2012). The parasite is arrested in early liver stage development, with each invading sporozoite giving rise to only a single hepatic parasite. Murine and non-human primate studies establish that the protective immunity induced by immunization with radiation attenuated sporozoites (RAS) is directed against the liver stage parasite and mediated primarily by $\mathrm{CD} 8^{+}$T cells and IFN- $\gamma$ (Schofield et al., 1987; Doolan and Hoffman, 2000; Tsuji, 2010; Weiss and Jiang, 2012). Recent studies have shown that protection in humans can be induced in a dose-dependent manner by intravenous but not intradermal immunization with radiation-attenuated, aseptic, purified, cryopreserved P. falciparum sporozoites (Epstein et al., 2011; Seder et al., 2013); five intravenous doses of 135,000 PfSPZ $(675,000$ sporozoites in total) were required to achieve sterile immunity in $6 / 6$ volunteers. $\mathrm{CD}^{+}$IFN- $\gamma$-producing $\mathrm{T}$ cells in the liver were implicated as the primary immune effector based on non-human primate studies showing that high frequencies of
$\mathrm{CD}^{+} \mathrm{T}$ cells could be induced by intravenous but not intradermal routes of immunization, and mouse studies showing that these cells could protect (Epstein et al., 2011). These data are consistent with an earlier proposal (Langhorne et al., 2008) that sporozoites injected intravenously can enter the liver within seconds and be processed and presented by liver-resident antigen-presenting cells for induction of host immunity, whereas sporozoites inoculated intradermally via mosquito bite may take minutes to hours to enter the liver; or might may be taken up by a different type of antigen presenting cell such as the skin-derived CD103 ${ }^{+}$dendritic cells (Bedoui et al., 2009).

Sterile immunity against Plasmodium sporozoite challenge can be also induced in mice by homologous immunization with infectious (live) wild type sporozoites while receiving a prophylactic regimen of chloroquine (Beaudoin et al., 1977; Orjih et al., 1982; Belnoue et al., 2004) or primaquine (Putrianti et al., 2009). This immunity is directed against the liver stage and mediated by $\mathrm{CD}^{+}$and $\mathrm{CD}^{+}{ }^{+} \mathrm{T}$ cells, but not antibodies (Belnoue et al., 2004; Roestenberg et al., 2009). More recently, this observation has been translated to humans with the demonstration that human subjects exposed three times to the bites of 10-15 P. falciparum infected mosquitoes under the cover of chemoprophylaxis (ChemoProphylaxis and Sporozoites, CPS-immunization; also known as infection-treatment-vaccination, ITV) were sterilely protected against subsequent challenge with $P$. falciparum sporozoites, but not $P$. falciparum-infected erythrocytes (Roestenberg et al., 2009; Bijker etal., 2013, 2014). This protection was sustained for up to 2 years (Roestenberg et al., 2011) and was dose dependent: complete protection was obtained in $4 / 5,8 / 9$, and $5 / 10$ volunteers immunized three times with bites from 15,10 , or 5 P. falciparum-infected mosquitoes, respectively, and CPS immunization is thus estimated to be about 20 times more efficient than RAS immunization (Bijker et al., 2014). Since chloroquine kills asexual blood stage parasites but not sporozoites or liver stage parasites, in the CPS-model parasite infection is aborted in the early phase of blood-stage infection allowing full liver-stage development of the parasite. Consequently, CPS immunization exposes the host to parasite antigens expressed in early and late liver stages as well as early blood stages (Bijker et al., 2013). However, the protective immunity appears to be directed primarily against the liver-stage of the parasite since CPS-immunized volunteers showed no evidence of protection against blood-stage challenge in vivo and IgG from CPS-immunized volunteers did not inhibit asexual blood-stage growth in vitro (Bijker et al., 2013). Moreover, this protection appears to be mediated by $\mathrm{T}$ cells since protected subjects had significantly higher proportions of $\mathrm{CD}^{+}{ }^{+} \mathrm{T}$ cells expressing the degranulation marker CD107a and $\mathrm{CD}^{+} \mathrm{T}$ cells producing granzyme $\mathrm{B}$ after in vitro restimulation with $P$. falciparum-infected red blood cells (Bijker et al., 2014), and antibodies to nine antigens representing different stages of the $P$. falciparum life cycle did not predict protection (Nahrendorf et al., 2014) even though CPS-immunization induced functional antibodies against P. falciparum sporozoites which could inhibit sporozoite traversal through hepatocytes and liver-stage infection (Behet et al., 2014). The antigenic targets of the CPS-induced $\mathrm{T}$ cell mediated are not yet known but this is under investigation. 
In a variation on the CPS approach, the induction of robust protective immunity by prophylactic administration of antibiotic drugs which specifically inhibit apicoplast biogenesis during exposure to intravenously or mosquito bite transmitted sporozoites was reported in the P. berghei murine model (Friesen et al., 2010). This approach was conceived to overcome limitations of ITV associated with drug resistance parasite populations. The correct choice of antibiotic in this model allows for continued liver-stage maturation and exponential expansion of attenuated liver-stage merozoites from a single sporozoite and subsequent release into the host peripheral circulation of merosomes (detached vesicles containing liver stage merozoites) which are incapable of infecting red blood cells, thereby halting the parasite life cycle prior to the asexual blood stage. This immunity appears to be targeted at the liver stage and mediated primarily by $\mathrm{CD}^{+} \mathrm{T}$ cells and IFN- $\gamma$. This strategy is distinct from the RAS model where each sporozoite gives rise to only a single attenuated liver-stage parasite, and the primaquine chemoprophylaxis model where liver-stage development is aborted before the onset of nuclear divisions (Putrianti et al., 2009), and the chloroquine chemoprophylaxis model which targets the early blood-stage (Belnoue et al., 2004; Roestenberg et al., 2009).

Another area of active investigation is genetically attenuated parasites (GAP) generated via targeted disruption of genes essential for liver-stage or blood stage development (Mueller etal., 2005). These have been comprehensively reviewed elsewhere (Butler etal., 2011; Matuschewski etal., 2011; NganouMakamdop and Sauerwein, 2013) and include GAPs that arrest development early $(\Delta \mathrm{p} 52 / \Delta \mathrm{p} 36, \Delta \mathrm{SAP} 1, \Delta \mathrm{SLARP})$ or later $(\Delta \mathrm{UIS} 3 / \mathrm{UIS} 4, \Delta \mathrm{E} 1 \mathrm{a}, \Delta \mathrm{E} 3, \Delta \mathrm{FABI}, \Delta \mathrm{FABB} / \mathrm{F}, \Delta \mathrm{FABZ}$, and $\triangle \mathrm{PKG})$ during liver stage development. In a first-in-human safety and immunogenicity clinical trial, 5/6 volunteers administered GAP sporozoites deleted of two P. falciparum pre-erythrocytic stage-expressed genes (P52 and P36) via mosquito bite did not develop blood stage parasitemia (Spring et al., 2013). However, the development of peripheral parasitemia in one volunteer showed that this double knockout GAP was incompletely attenuated. Although no breakthrough blood infections were observed in a study evaluating the P. yoelii $\Delta \mathrm{p} 52 / \Delta \mathrm{p} 36$ GAP (Labaied et al., 2007), others observed developing $P$. berghei or $P$. falciparum liver stages in vitro culture with the respective $\Delta \mathrm{p} 52 / \Delta \mathrm{p} 36$ GAPs and breakthrough blood infections in P. berghei $\Delta \mathrm{p} 52 / \Delta \mathrm{p} 36 \mathrm{GAP}$ immunized mice, showing that the $\Delta \mathrm{p} 52 / \Delta \mathrm{p} 36$ GAP was not adequately attenuated (Annoura et al., 2012). A minimal set of screening criteria has been proposed to assess the adequacy of genetically attenuation before advancing candidate GAPs into further clinical development (Annoura et al., 2012). Most recently, a triple gene deleted GAP (Pf $\Delta \mathrm{p} 52 / \Delta \mathrm{p} 36 / \Delta$ sap 1 ) had been shown to be completely attenuated in a humanized mouse model (Mikolajczak et al., 2014).

Vaccination with chemically attenuated parasites is also being pursued. In the original studies in mice, chemical attenuation of $P$. yoelii or P. berghei sporozoites with the DNA sequencespecific alkylating agent centanamycin conferred sterile immunity in vivo following one to three intravenous doses (50/20/20K) of centanamycin-treated $P$. yoelii or $P$. berghei sporozoites (Purcell et al., 2008a,b). The level of protection, parasite-specific antibodies, and IFN- $\gamma$-producing $\mathrm{CD} 8^{+} \mathrm{T}$ cell responses induced by chemically attenuated sporozoites (CAS) were similar to those induced by RAS. In the blood stage, Good et al., (2013) have recently reported that a single immunizing dose of $10^{6} \mathrm{P}$. chabaudi parasitized red blood cells chemically attenuated with centanamycin could protect against challenge with $10^{5}$ homologous or heterologous (P. vinckei and P. yoelii) parasites in a CD4 $4^{+}$ T cell dependent manner (Good et al., 2013). Chemically attenuated P. falciparum parasitized red blood cells are currently being evaluated in the clinic (Good, personal communication).

Another whole organism based strategy directed at the blood stage of the parasite life cycle was designed to induce $\mathrm{T}$ helper 1 (Th1) cell mediated immunity in the absence of antibodies by immunizing with subpatent ultra-low dose parasitized erythrocytes followed by drug treatment (Pombo et al., 2002). This built on observations that parasites in high density could cause apoptosis of parasite-specific T cells (Hirunpetcharat and Good, 1998; Xu et al., 2002). Good et al. (2013) showed that malaria-naive humans deliberately infected four times with approximately 30 viable parasitized red blood cells followed by drug treatment developed robust $\mathrm{T}$ cell responses in the absence of antibody which prevented parasite growth in three of four individuals and delayed the onset of parasite growth which remained subpatent in the fourth individual (Pombo et al., 2002). Efficacy against a higher dose challenge postimmunization was not assessed, and the contribution of residual drug to this protection could not be excluded. This ultra-low dose immunization approach has not yet been repeated in humans or mice. However a subsequent study in the P. chabaudi model demonstrated that three intravenous infections with a relatively high dose of 100,000 P. chabaudi infected erythrocytes followed by drug cure after $48 \mathrm{~h}$ and before microscopic patency could protect mice against a 10 -fold higher $\left(10^{6}\right)$ parasite challenge; mice had robust cell-mediated immune responses and antibodies to merozoite antigens but variant-specific antibodies were not detectable (Elliott et al., 2005).

These proof-of-concept studies with whole organism based vaccines show that experimental induction of sustained protective immunity to Plasmodium spp. parasites is possible.

\section{MALARIA VACCINE STRATEGIES}

Evidence that immunity can be induced experimentally or acquired naturally with age and/or exposure suggests two fundamental approaches to vaccine development (Good and Doolan, 2010):

(1) Induce robust immune responses against a selected panel of antigens recognized as immunodominant in the context of natural infection.

(2) Induce a broad immune response against a large number of parasite antigens not necessarily recognized as immunodominant in the context of natural infection in order to mimic the immunity induced by the whole parasite.

Until recently, almost all malaria vaccine efforts have been directed at the former approach. Most of these subunit efforts have targeted only a very small number of target antigens, focusing almost exclusively on CSP for the pre-erythrocytic stage and MSP1 and AMA1 for the blood stage (Schwartz et al., 2012; World 
Health Organization [WHO], 2014b) and investigating a variety of vaccine delivery systems. A major emphasis has been on purified recombinant proteins formulated with adjuvant, but viral vectored approaches have become of increasing interest, particularly for the pre-erythrocytic stage where induction of parasite-specific $\mathrm{T}$ cell responses is desirable. These have been reviewed extensively elsewhere (Bruder etal., 2010; Crompton etal., 2010b; Anders, 2011; Schwartz et al., 2012; Birkett et al., 2013). However, despite extensive efforts throughout the world spanning many decades and in contrast to the immunity induced by experimental immunization with variations of whole organism based vaccines, candidate subunit vaccines against malaria have been poorly efficacious (Schwartz et al., 2012; World Health Organization [WHO], 2014b). Indeed, it is not surprising that a vaccine based on a single antigen is unlikely to confer solid protection against a complex multi-lifecycle stage parasite expressing thousands of proteins that has co-evolved with the human host for millennia.

This marked lack of success in single-antigen subunit based vaccines, combined with the recognition that an effective malaria vaccine will likely need to be a multi-stage multi-immune response vaccine (Doolan and Hoffman, 1997) given the challenges described above, has caused a resurgence of interest in whole organism vaccine approaches, intended to reproduce the protective immunity induced by exposure to the parasite in experimental challenge models or naturally in the field. However, a number of challenges are associated with whole organism based vaccine strategies (Menard, 2005; Ballou and Cahill, 2007; Anders, 2011). Specific concerns with GAPs include potentially inadequate attenuation, as already demonstrated with the $P f \Delta 52 / \Delta$ p 36 GAP in the only human trial to date (Spring et al., 2013), and reversion to virulence of a parasite that has co-evolved with the human host for millennia if it is genetically modified in only one or a few regions of its genome. Other concerns include logistical challenges associated with manual dissection of sporozoites, route of administration, loss of viability upon cryopreservation, and cold-chain requirements. Additionally, antigenic variability of the parasite means that robust cross-protection from a single strain product is essential. Thus, although promising results have been obtained in preclinical models, it remains to be seen whether the many technical, logistical, and regulatory hurdles associated with large-scale production and field deployment of live-attenuated parasites can be overcome.

Even if these technical, logistical, and regulatory challenges can be overcome, a key question is whether whole parasite approaches will induce optimal immunity. Those approaches are essentially similar to the classical "identify, isolate, and inject" approach pioneered in the late 17th century by Edward Jenner, which has proved successful with a wide range of bacterial and viral pathogens, but not yet any parasitic pathogens or any chronic diseases (reviewed in Doolan etal., 2014). This could be attributed to the complexity of parasites as compared to viruses and bacteria, with larger genomes and multiple intracellular and extracellular life cycle stages. Additionally, it is now well established that microbial pathogens have evolved complex and efficient ways of counteracting and evading innate and adaptive immune mechanisms of the host (Zepp, 2010). Thus, logically, robust immunity against such pathogens would not be induced by strategies using the whole pathogen intended to mimic experimentally that immunity induced by natural exposure. Rather, effective vaccination would require that we do better than nature, by inducing responses that are quantitatively and/or qualitatively different immune response to that induced by natural infection.

Inherent in this approach is the cumulative effect of multiple potentially low level immune responses directed against a number of antigens which may or may not be dominant parasite antigens, which together exceed a response threshold sufficient to protect. We proposed this "threshold of immune response concept" over 15 years ago (Doolan and Hoffman, 1997). Specifically, we proposed that the intensity of an immune response will be determined by the sum of a number of signals received by a T cell (or B cell) with the appropriate receptor, and that although a single antigen (with one or more target epitopes) could be sufficient to generate a protective immune response if it is appropriately presented to the immune system, a wide repertoire of specificities at the epitope level (more antigens) should increase the probability of collectively inducing a protective host immune response. Experimental evidence that responses to a given antigen following protective immunization in mice with RAS are not as high as antigen-specific responses induced by vaccination with antigen-specific peptides, recombinant protein or live vectors which nonetheless fail to protect validates this concept. In an elegant series of studies in the $P$. berghei model, Harty and colleagues were able to define a threshold frequency of $\mathrm{CD}^{+}{ }^{+} \mathrm{T}$ cells that predicted long-term sterile immunity against sporozoite challenge, and showed that an extremely high frequency of $\mathrm{CD}^{+}{ }^{+} \mathrm{T}$ cells (exceeding $8 \%$ of all circulating $\mathrm{CD}^{+} \mathrm{T}$ cells in BALB/c mice and $19 \%$ in outbred mice) was required for both a single-antigen subunit vaccination (Schmidt et al., 2008) and RAS immunization (Schmidt et al., 2010); this level greatly exceeding the number of memory $\mathrm{CD}^{+} \mathrm{T}$ cells required for resistance to other pathogens.

A similar requirement for a protective threshold of antibody production for parasite clearance following lethal challenge has been demonstrated in a $P$. chabaudi model using MSP1-specific transgenic $\mathrm{CD}^{+}{ }^{+} \mathrm{T}$ cells in immunodeficient mice, where levels of MSP1-specific antibody and the speed of their production correlated with the time of resolution of infection (Stephens et al., 2005). In humans, in the field, a broad repertoire of antibody responses to multiple antigens has been associated with protection from clinical malaria (Osier et al., 2008; Crompton et al., 2010a).

These data suggest that to induce optimal protection against malaria, vaccination with the whole parasite is not required, and would likely be suboptimal. Rather, vaccination with only key components of the parasite that have been rationally selected in the context of relevant biological function would be preferable.

\section{NOT ALL ANTIGENS ARE EQUAL}

It is now generally recognized that not all antigens, or epitopes within a given antigen, are equal in the context of host immunity. The phenomenon whereby immune responses are mounted against only one or a few of the entire repertoire of peptide epitopes expressed on a given antigen, or antigens expressed by a given pathogen, is termed immunodominance (Sercarz et al., 1993; Akram and Inman, 2012). In theory, any of the proteins expressed by the parasite genome may be a target of protective 
immune responses. However, many proteins expressed by the parasite genome do not elicit immune responses, and for many if not most of the subset which do elicit immune responses, the response is not protective. Also in theory, a robust and effective immune response directed against an accessible dominant target would be highly successful in eliminating the pathogen from the host. However, as noted above, in many cases immune evasion strategies have evolved to allow the pathogen to escape the protective host response. Factors that could influence immunodominance, and their importance in protection, have not been investigated in the context of a complex pathogen, although some hypotheses exist (Doolan et al., 2014).

Although immunization with whole organisms preferentially induces responses against immunodominant epitopes, responses to subdominant $\mathrm{T}$ cell epitopes can contribute to controlling infection (Friedrich et al., 2007; Kloverpris et al., 2009; Ruckwardt et al., 2010). Also, the ability to focus the immune response away from dominant antigens or epitopes and towards subdominant antigens or epitopes could be of value in chronic diseases where T cells directed against the immunodominant antigens or epitopes might be anergic but $\mathrm{T}$ cells specific for non-dominant epitopes might be reactive. Translating this to infectious diseases where the development of effective vaccines based on immunodominant antigens has thus far not been successful (Good and Doolan, 2010), one could speculate that the critical targets of protective immunity may be those that are not dominant in the context of the whole organism.

In support of this, although the CSP is the dominant sporozoite surface protein and represents a target of immune responses induced by immunization with radiation attenuated Plasmodium sporozoites, those responses are much weaker than responses induced by CSP-based subunit vaccines, and responses are also directed against other non-CSP antigens (Doolan et al., 1997, 2000, 2003; Kumar et al., 2006; Gruner et al., 2007; Trieu et al., 2011). Notably, sterile $\mathrm{CD}^{+}$T-cell mediated immunity to sporozoite challenge could be induced by immunization with RAS in JHT transgenic mice that were tolerant to CSP, so this protection was directed against non-CSP antigens (Kumar et al., 2006). Sterile protection could be also induced by RAS or CPS immunization with transgenic $P$. berghei parasites in which the endogenous CSP was replaced by that of $P$. falciparum or $P$. yoelii, respectively, despite the absence of immune responses specific to the CSP expressed by the parasite used for challenge (Gruner et al., 2007; Mauduit et al., 2010). Also, human volunteers protected by immunization with RAS did mount $\mathrm{CD}^{+}$and $\mathrm{CD} 4^{+} \mathrm{T}$ cell and antibody responses to CSP but those responses were similar to, or lower than, those in immunized volunteers who were not protected against sporozoite challenge indicating that the RASinduced protective immune responses are directed predominantly against non-CSP antigens (Doolan et al., 1997, 2000, 2003; Trieu et al., 2011). Recently, using protein microarrays, we have identified a signature of 19 mostly uncharacterized antigens which is strongly associated with RAS-induced protective immunity; reactivity to any individual antigen did not correlate with protection (Trieu et al., 2011).

Accumulating experimental data in preclinical and clinical studies of malaria thus indicate that in fact not all antigens are equal, that antigen selection is important, and that it is the cumulative response to a number of key antigens that is important, rather than a dominant response to a single antigen.

\section{GENOME-BASED VACCINE DESIGN}

The identification within the hierarchy of antigens (or epitopes) expressed by the pathogen that are targets of protective immune responses and that will stimulate effective immunity against that pathogen is a key component of rational vaccine design (Rueckert and Guzman, 2012). Cutting-edge technologies and screening strategies to mine genomic sequence information for state-ofthe-art rational vaccine design, as well as genome-based rational vaccine design strategies, and recently reviewed elsewhere (Doolan et al., 2014; Schussek et al., 2014). The challenge, then, is how to select the key targets since there is no algorithm that can be applied to identify the important antigens and epitopes. Advances in the genomic era offer great potential, particularly when the genome of the target pathogen is large, and large-scale genomic, proteomic and transcriptomic datasets provide valuable resources to mine for antigen discovery.

In the case of malaria, the recent availability of large-scale genomic (Table 1), proteomic (Table 2), transcriptomic (Table 3) and comparative data from $P$. falciparum and other Plasmodium species provides an unprecedented opportunity to identify key targets antigens of protective immunity amongst the large repertoire of antigens expressed by the whole parasite. Since 2002, the genomes of seven Plasmodium parasites have been published, including that for the two major human parasites ( $P$. falciparum, $P$. vivax) (Gardner et al., 2002; Carlton et al., 2008; Pain et al., 2008); two non-human primate parasites ( $P$. knowlesi, P. cynomolgi; Pain et al., 2008; Tachibana et al., 2012), and three murine parasites ( $P$. yoelii 17XNL, P. berghei, P. chabaudi; Carlton et al., 2002; Hall et al., 2005). Draft complete genomes are also available for the avian malaria parasite $P$. gallinaceum, non-human primate parasite $P$. reichenowi Denni, the lethal murine parasite $P$. yoelii $Y M$, and three different strains of $P$. falciparum (HB3,Dd2 and IT; Table 1). Partial genome sequence is also accessible for 21 isolates of $P$. falciparum and four isolates of $P$. vivax from geographically distinct areas of the world, as well as low coverage draft genomes for the other two parasites infecting humans, $P$. malaria and $P$. ovale. With the advent of next-generation sequencing technology, the sequencing of genomes for an additional 105 Plasmodium species/strains/isolates have been proposed by the malaria community ${ }^{6}$. These parasites include $50 \mathrm{P}$. falciparum field isolates collected from patients in East Africa, America, and Asia; 24 P. falciparum parasites representing both contemporary or historical parasite strains, including strains used in drug and vaccine trials; $16 P$. vivax isolates from Africa, America, and Asia; four nonhuman primate parasites (P. reichenowi, P. cynomolgi, P. inui, P. coatneyi, $P$. fragile); and complete sequence and closure of three murine parasites ( $P$. chabaudi, $P$. yoelii, and $P$. berghei and two avian and reptile parasites ( $P$. relictum and $P$. mexicanum). For some of these parasites, a first partial assembly is already available (Table 1).

${ }^{6}$ http://www.genome.gov/pages/research/der/pathogensandvectors/plasmodium whitepaperv8.pdf 


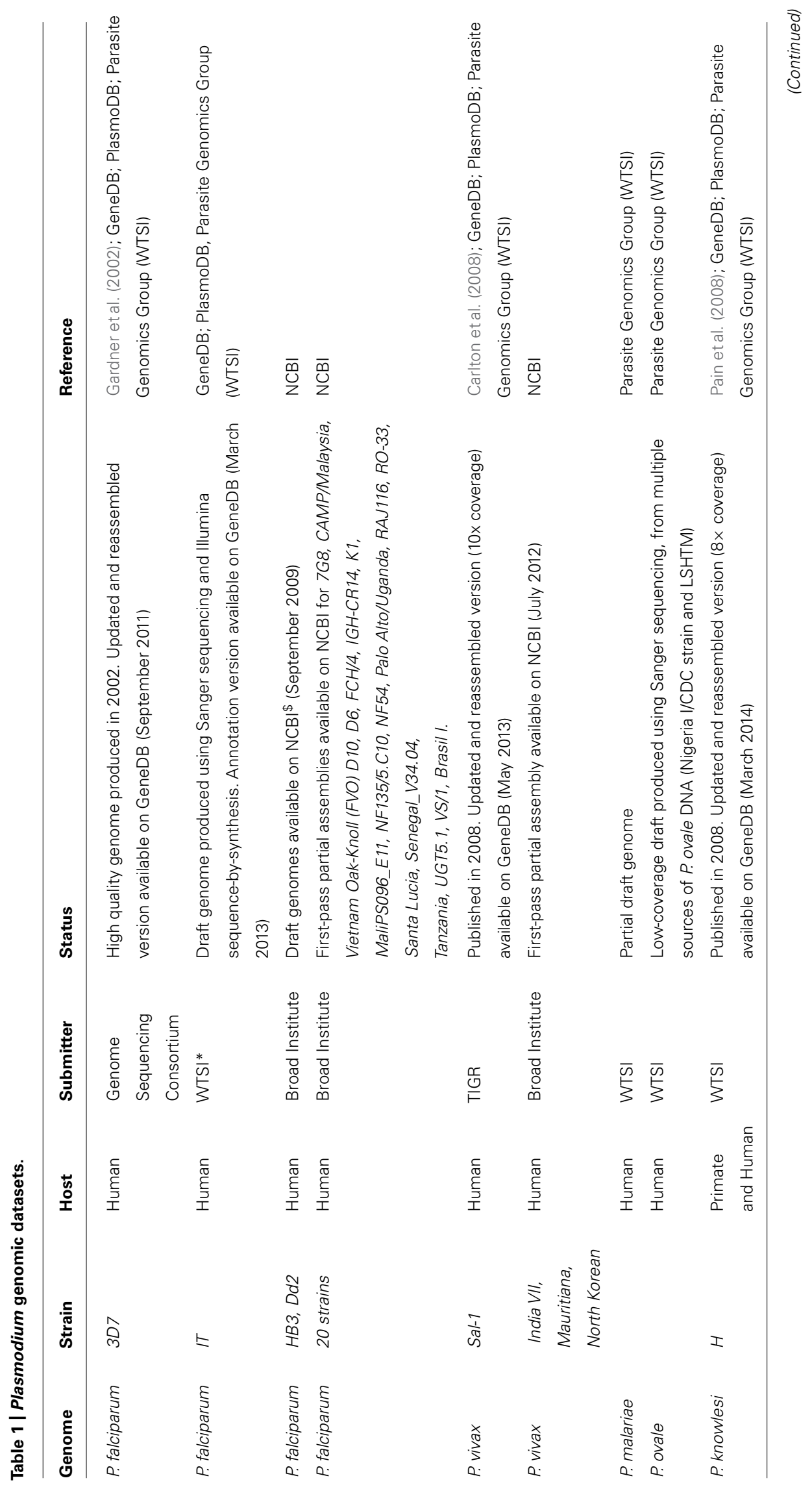




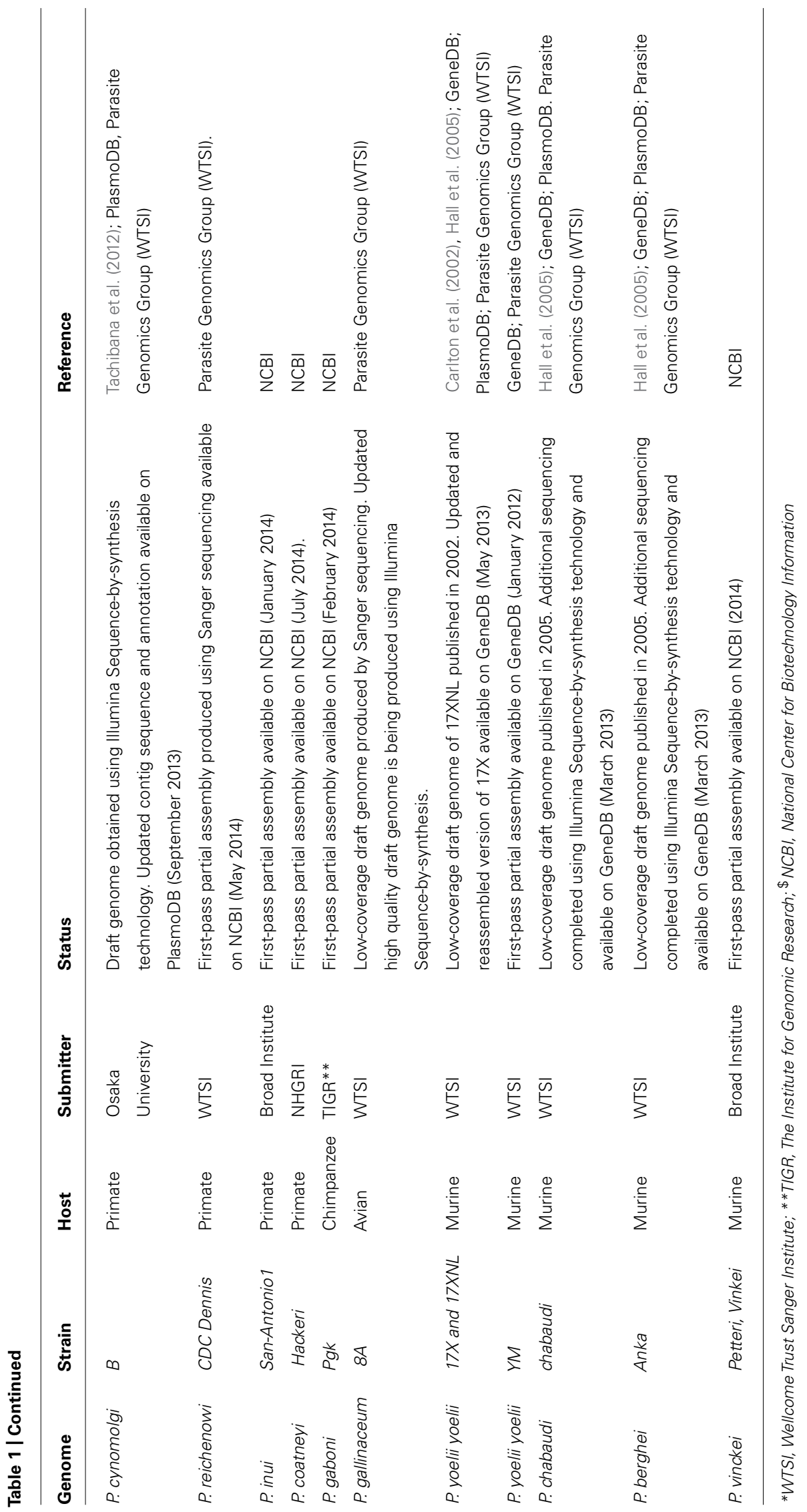




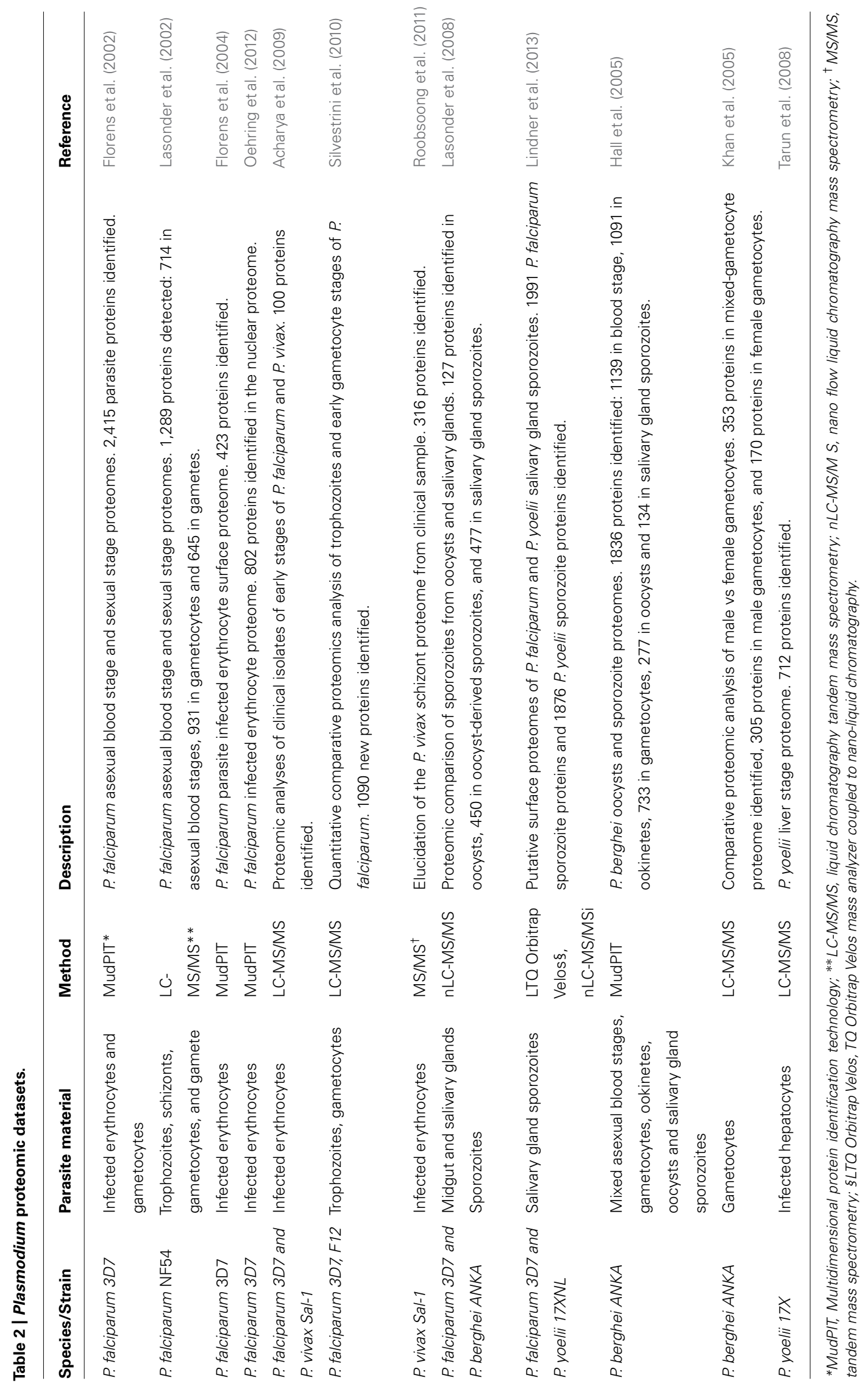




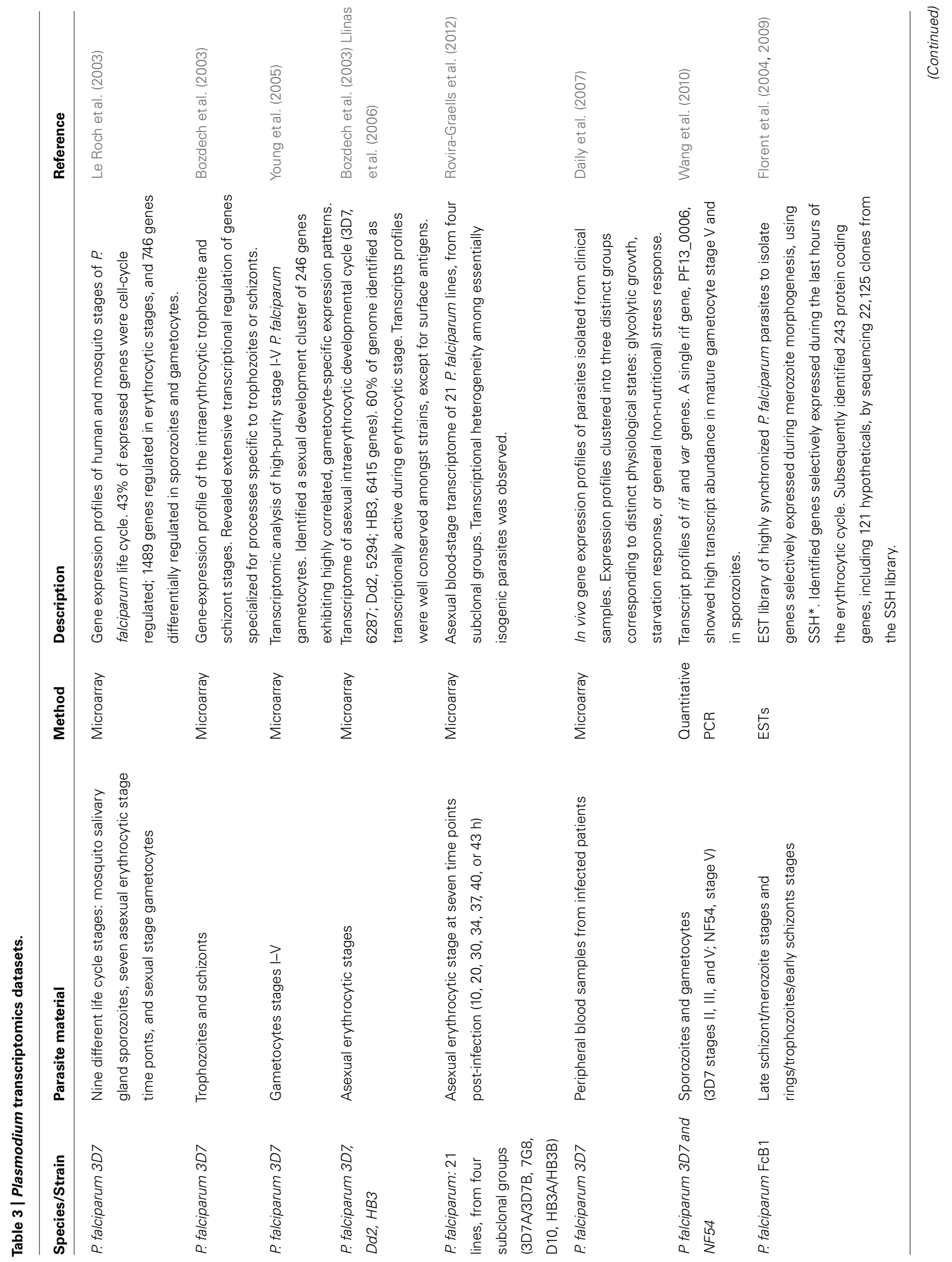




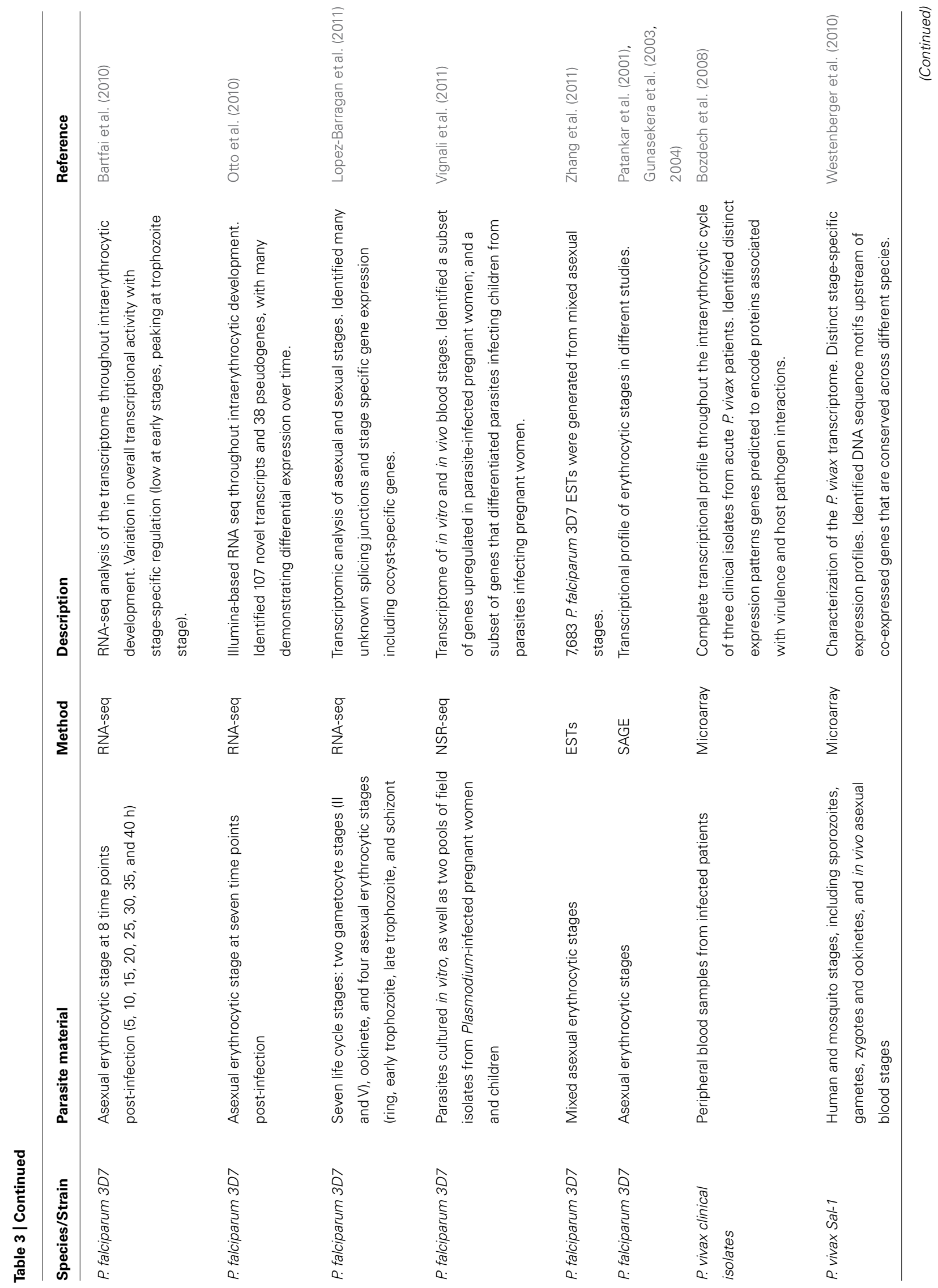




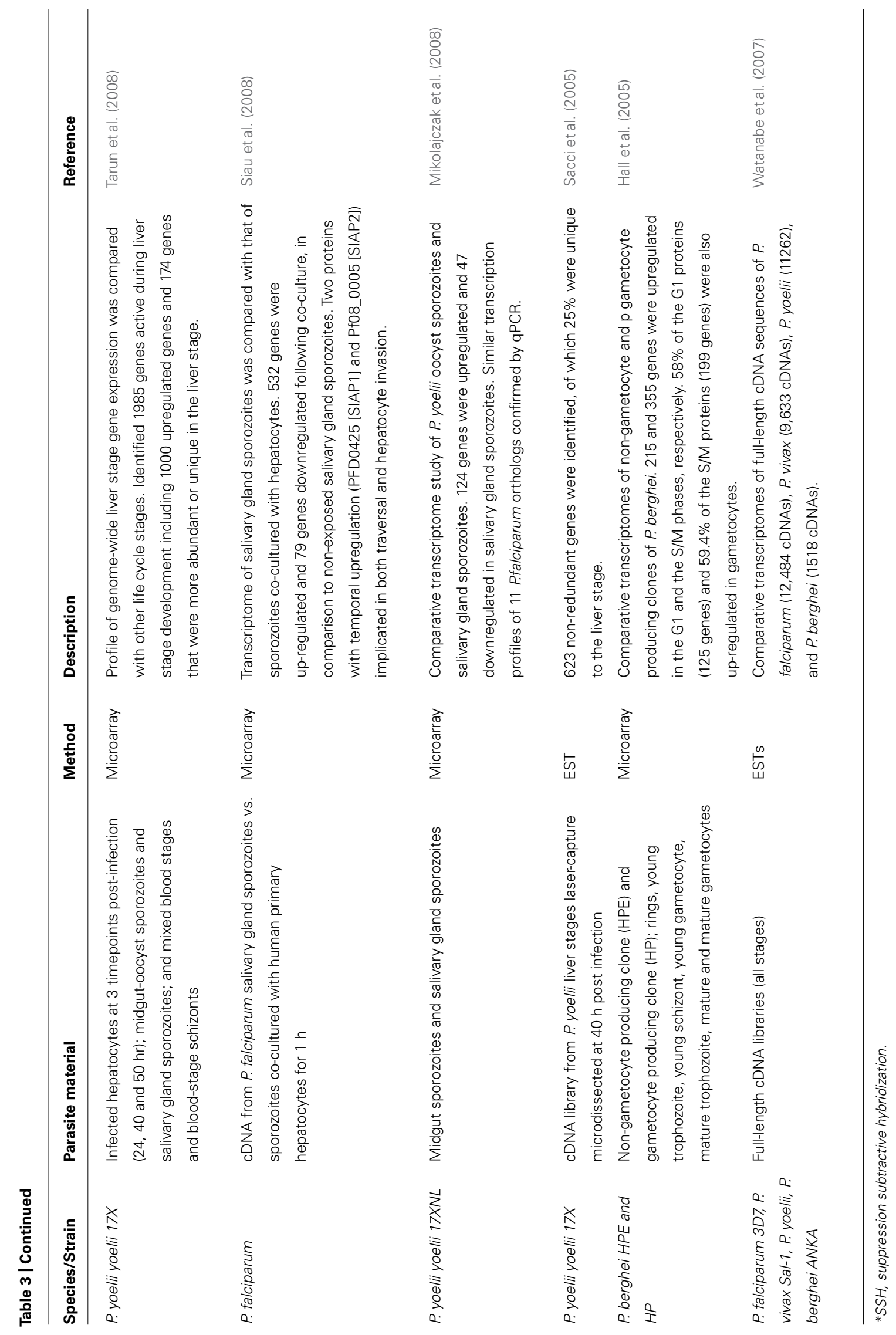


In addition to this genomic data, the rapid development of high throughput technologies for profiling the transcriptome, proteome, metabolome, and interactome, including capillary liquid chromatography, tandem mass spectrometry (LC-MS/MS), Multidimensional Protein Identification Technology (MudPIT), microarray DNA chip, yeast two-hybrid (Y2H) screening and most recently RNA seq and NSR-seq (Winzeler, 2006) can be applied for the rational identification of potential vaccine candidate antigens.

In early studies, proteomes of four stages of the $P$. falciparum parasite life cycle (sporozoites, merozoites, trophozoites, and gametocytes) were revealed by MudPIT (Florens et al., 2002) as well as the proteome of the asexual blood stages (trophozoites and schizonts) and sexual stages (gametocytes and gamete) by LC-MS/MS analysis (Lasonder et al., 2002). This P. falciparum sporozoite proteome included a total of 1048 proteins of which almost half (49\%) were unique to this stage. The proteomes of $P$. berghei oocysts and sporozoite were subsequently defined by MudPIT in 2005, resulting in the identification of 1836 proteins (Hall et al., 2005). Recently, nano-liquid chromatography (nanoLC) coupled high-resolution MS was applied to profile the proteome of highly purified salivary gland sporozoites from $P$. falciparum and P. yoelii, identifying a total of 1991 P. falciparum sporozoite proteins and 1876 P. yoelii sporozoite proteins (Lindner et al., 2013). The liver stage proteome was defined in the rodent host $P$. yoelii by LC-MS/MS resulting in the detection of 712 proteins in the liver stage schizont proteome, with 174 of them more abundant and/or detected only in the liver stage (Tarun et al., 2008).

A study of $P$. falciparum infected erythrocytes, fractionated through biotin-streptavidin interaction and analyzed by MudPIT, identified 164 proteins of the 423 proteins that were enriched the in biotin-labeled fractions and thus considered surface proteins. Among these were known secreted proteins, such as Exp-1 and Exp-2, and rhoptry proteins (RAP-1, -2, and -3, RhopH-2 and -3; Florens et al., 2004). Another LC-MS/MS study of early stages from $P$. falciparum clinical isolates detected 88 P. falciparum proteins in the peripheral circulation (Acharya et al., 2009). More recent analyses on the proteome of asexual trophozoites, early gametocytes, and mature gametocytes from in vitro culture by high accuracy (LC MS/MS) have identified over new 1000 parasite proteins, not previously identified (Silvestrini et al., 2010). Most recently, proteomic analysis by MudPIT of $P$. falciparum nuclei of ring, trophozoite and schizont stages has elucidated the nuclear proteome of $P$. falciparum during intra-erythrocytic development, consisting of over 800 proteins (Oehring etal., 2012).

Elucidation of the gametocyte sex-specific proteomes of $P$. falciparum by LC-MS/MS resulted in the identification of 305 unique proteins in the male gametocyte proteome and 170 unique proteins in the female gametocyte proteome (Khan et al., 2005). The identification of sex-specific proteins has brought new insight in understanding the role of these proteins during the sexual differentiation and thus proving the basis for identifying targets for the interruption of transmission, either by drugs or vaccines.

Other comparative LC MS/MS proteomic studies of $P$. falciparum and $P$. berghei have identified novel proteins in the pre-erythrocytic stages of the Plasmodium life cycle: 127 proteins in the oocyst proteome, 450 proteins in oocyst-derived sporozoites and 477 proteins in salivary gland sporozoites, for a total of 728 Plasmodium proteins, of which 250 were exclusively detected in the oocyst/sporozoite stages when compared to the $P$. falciparum blood stage proteome (Lasonder et al., 2008).

More recently, the proteome of $P$. vivax asexual schizonts has been defined by analyzing fresh parasite isolates from patients exposed to $P$. vivax by tandem MS/MS (Roobsoong et al., 2011).

Complementing the proteomic analyses, a number of transcriptomics studies have been undertaken, ranging from analysis of gene transcription using random clones selected from genomic DNA libraries to more recent global expression transcription profile using oligonucleotide microarray, RNA-seq or NSR-seq. Transcriptomic data are now available from multiple life-cycle stages or gene knock-out mutants of $P$. falciparum and $P$. berghei as well as multiple stages of $P$. yoelii (mosquito, erythrocytic and liver stages; Table 3). Specifically, transcriptomic data available for $P$. falciparum include genome-scale transcriptomic analyses of nine different life cycle stages (3D7 strain) including salivary gland sporozoite, early and late ring stage, early and late trophozoite, early and late schizont, merozoite, and gametocyte stages (Le Roch et al., 2003); the intraerythrocytic trophozoite and schizont stages (Bozdech et al., 2003); the intraerythrocytic developmental cycle of P. falciparum HB2, Dd2, and 3D7 strains (Llinas et al., 2006); as well as 21 other $P$. falciparum lines from four subclonal groups (3D7A/3D7B, 7G8, D10, HB3A/HB3B) during the asexual intraerythrocytic developmental cycle at seven time points post-infection $(10,20,30,34,37,40$, or 43 h; Rovira-Graells et al., 2012) plus analysis of parasites derived directly from blood samples from 43 infected patients (Daily et al., 2007). The transcriptome of high-purity stage I-V P. falciparum gametocytes is also available (Young et al., 2005), as well as the transcript profiles of rif and var genes at different stage of gametocytogenesis (Wang et al., 2010).

In addition to genome-wide gene expression studies, RNA-seq analysis of the P. falciparum transcriptome is available for multiple time points during the intraerythrocytic developmental cycle (Bartfai et al., 2010; Otto et al., 2010; Lopez-Barragan et al., 2011); and two gametocyte stages (Lopez-Barragan et al., 2011). The transcriptional profile of two pools of field isolates from malariainfected pregnant women and children has been also determined by NSR-seq (Vignali et al., 2011).

For $P$. vivax, transcriptomic data include genome-scale transcriptomic analyses throughout the intraerythrocytic cycle of three distinct $P$. vivax isolates (Bozdech et al., 2008) as well as sporozoites co-cultured with hepatocytes (Siau et al., 2008); and sporozoites, gametes, zygotes, and ookinetes, and asexual blood stages obtained from infected patients (Westenberger et al., 2010).

Plasmodium yoelii gene expression data are available for oocyst sporozoites and salivary gland sporozoites (Mikolajczak et al., 2008); three time points during the liver stage $(24,40$, and $50 \mathrm{~h}$ post-infection), two time points during the mosquito stage (midgut-oocyst sporozoites and salivary gland sporozoites; Tarun et al., 2008), and two intraerythrocytic stages (Tarun et al., 2008). P. berghei expression data are available for rings, young 
trophozoites, young schizonts, and mature schizonts (Hall et al., 2005).

Other transcriptomic datasets include EST data from cDNA libraries of $P$. falciparum (12,484 cDNA sequences), $P$. vivax (9,633 cDNAs), $P$. yoelii (11,262 cDNAs), and $P$. berghei (1,518 cDNAs) (Watanabe et al., 2007; Tarun et al., 2008), as well as 7,683 P. falciparum 3D7 ESTs generated from mixed asexual stages and SAGE data (Patankar et al., 2001; Gunasekera et al., 2003, 2004).

These genome-wide genomic, proteomic, and transcriptomics analyses (Tables 1-3) have revealed potential antigens expressed in the sporozoite and intrahepatic stages and novel proteins on the surface of malaria-infected erythrocytes that may play a role in pathogenesis and immunity, and that may represent potential new vaccine candidates.

Several novel parasite surface antigens have been discovered (Florens etal., 2002, 2004; Lasonder et al., 2002; Le Roch et al., 2004; Sam-Yellowe et al., 2004) but, so far, this wealth of data has yielded few new vaccine targets (Table 4; Duffy et al., 2012). In our opinion, translation of this wealth of information from the large-scale genomic, proteomic, and transcriptomic datasets into practical application, such as the identification of promising new target antigens for vaccine development, requires integrating this knowledge with functional outputs such as biologically relevant immune responses. Thus, in our laboratory, we are pursuing immunomics-based approaches which integrate the disciplines of genomics and immunology using biological samples from humans or animals with immunity to the disease of interest to identify the subset of pathogen-derived proteins or their epitopes that are recognized by the host immune system (Klysik, 2001; Doolan, 2011). No vaccines derived from immunomics have yet reached the stage of clinical testing but a number of promising candidate antigens have been identified by us in the malaria model using antibody based (Doolan et al., 2008; Crompton et al., 2010a; Trieu et al., 2011) or T-cell based (Doolan et al., 2003; Doolan, 2011). We are using peripheral blood mononuclear cells ( $\mathrm{T}$ cells) and plasma/sera (antibodies) from individuals experimentally immunized with RAS or CPS or naturally exposed to malaria for proteome-wide immune screening assays using clinically relevant

Table 4 | Plasmodium antigens identified from genome-based datasets.

\begin{tabular}{|c|c|c|c|}
\hline Antigen & Model & Main finding & Reference \\
\hline Ag2/CelTOS & $\begin{array}{l}\text { P. falciparum RAS T cell screening; } \\
P \text {. yoelii and } P \text {. berghei murine } \\
\text { immunization/challenge }\end{array}$ & $\begin{array}{l}\text { One of four highly reactive } P \text {. falciparum proteins } \\
\text { identified by } T \text { cell based screening of } 27 \text { putative } \\
\text { proteins with RAS immunized volunteers; } \\
\text { conferred cross-species protection against } P \text { yoelii } \\
\text { and } P \text {. berghei sporozoite challenge. }\end{array}$ & $\begin{array}{l}\text { Doolan (in preparatior } \\
\text { Doolan et al. (2003), } \\
\text { Bergmann-Leitner et } \\
\text { (2010) }\end{array}$ \\
\hline $\begin{array}{l}\text { Thirty-four pre-erythrocytic } \\
\text { antigens }\end{array}$ & $P$ yoelii murine immunization/challenge & $\begin{array}{l}\text { Only three antigens (P33p[Py52] [PY01340], Ag2 } \\
\text { [PyCelTOS], and Ag5[PY00419] elicited CD8+ T } \\
\text { cell responses but none conferred protection. }\end{array}$ & Mishra et al. (2011) \\
\hline $\begin{array}{l}\text { PY03011, PY03424, and } \\
\text { PY03661: pre-erythrocytic } \\
\text { antigens }\end{array}$ & $P$ yoelii murine immunization/challenge & $\begin{array}{l}\text { The combination of the three antigens (but not } \\
\text { individual antigens) conferred sterile protection } \\
\text { against } P \text { yoelii sporozoite challenge in a high } \\
\text { proportion of mice. }\end{array}$ & Limbach et al. (2011) \\
\hline $\begin{array}{l}\text { PyTmp21(PY06414): } \\
\text { pre-erythrocytic antigen }\end{array}$ & $P$ yoelii murine immunization/challenge & $\begin{array}{l}\text { PyTmp21 elicited functional immunity that } \\
\text { significantly reduced liver stage parasite burden } \\
\text { following } P \text {. yoelii sporozoite challenge. }\end{array}$ & Chen etal. (2014) \\
\hline $\begin{array}{l}\text { PbS20 and PbTRAP } \\
\text { pre-erythrocytic antigens }\end{array}$ & P. berghei murine immunization/challenge & $\begin{array}{l}\text { Systematically evaluated } \mathrm{H}(2 \mathrm{~b}) \text {-restricted peptides } \\
\text { predicted from genome-wide analysis, and } \\
\text { identified two epitopes as targets of } \mathrm{CD}^{+} \mathrm{T} \text { cells } \\
\text { induced by whole parasite vaccines; } \mathrm{CD}^{+} \mathrm{T} \text { cells } \\
\text { specific for the PbTRAP epitope but not the PbS20 } \\
\text { epitope inhibited liver stage parasite development } \\
\text { in vivo }\end{array}$ & Hafalla et al. (2013) \\
\hline PyTAM: blood-stage antigen & $P$ yoelii murine immunization/challenge & $\begin{array}{l}\text { PYTAM elicited functional immunity that conferred } \\
\text { significant protection against mortality from lethal } \\
\text { P. yoelii challenge infection. }\end{array}$ & Cherif etal. (2014) \\
\hline $\begin{array}{l}\text { Py01157: sexual and sexual } \\
\text { stage antigen }\end{array}$ & $P$ yoelii murine immunization/challenge & $\begin{array}{l}\text { Py01157: conferred partial protection against } \\
\text { challenge with non-lethal } P \text {. yoelii } 17 \mathrm{XNL} \text {, but not } \\
\text { against challenge with lethal } P \text {. yoelii } 17 \mathrm{XL} \text {. }\end{array}$ & Zhang etal. (2012) \\
\hline
\end{tabular}


selection criteria to identify the key antigens and their epitopes recognized by recall Plasmodium-specific immune responses in protective human models. Data generated to date establish proofof-concept for both $\mathrm{T}$ cell and antibody based approaches to identify from genomic datasets the subset of antigens and epitopes which represent promising candidates for next generation malaria vaccine development (Doolan, 2011; Matuschewski et al., 2011; Doolan et al., 2014; Table 4).

In the initial proof-of-concept study (Doolan et al., 2008) for the application of protein microarray technology for antigen discovery, a protein microarray with 250 pre-erythrocytic $P$. falciparum proteins were probed with sera from individuals with sterile immunity or no immunity against experimental challenge following vaccination with radiation-attenuated $P$. falciparum sporozoites, partial immunity acquired by natural exposure, or no previous exposure to $P$. falciparum, to identify 72 highly reactive P. falciparum antigens. Subsequently, a larger protein microarray containing 2,320 P. falciparum proteins fragments (corresponding to 1200 P. falciparum proteins, $\sim 25 \%$ of the proteome) was screened with plasma from clinically divergent groups of individuals immunized with $P$. falciparum irradiated sporozoites and identified a signature of 16 antigens strongly associated with RAS-induced sterile protective immunity; responses to any individual antigen were not associated with protection (Trieu et al., 2011). Using this same protein microarray, plasma from children and young adults naturally exposed to malaria in an endemic area of Mali were screened and 46 novel proteins significantly associated with a reduction of re-infection in the subsequent malaria season were identified (Crompton et al., 2010a). One $P$. falciparum antigen identified by $\mathrm{T}$ cell based screening of RASimmunized volunteers (Doolan etal., 2003), known as Ag2 or CelTOS (Kariu etal., 2006), has been shown to be a target of cross-species protection in the murine model (Bergmann-Leitner et al., 2010). Another P. yoelii antigen, PyTmp1, could induce immune responses that reduced liver stage parasite burden in vivo (Chen et al., 2014), and three other $P$. yoelii antigens in combination (but not individually) could induce sterile immunity in a high proportion of immunized mice (Limbach et al., 2011). Two P. yoelii blood stage antigens, PyTAM and Py01157, have been also associated with partial protection in the murine model (Zhang et al., 2012; Cherif et al., 2014; Table 4). These data support the potential of genome-based antigen discovery. Assessment of the protective capacity of promising new antigens discovered by genome-based screening is currently a bottleneck in the preclinical development pipeline. Development and refinement of a high throughput screening system would overcome this obstacle and allow the development of a rationally designed genome-based vaccine against malaria.

\section{CONCLUSION}

We advocate a modern genome-based approach to rational vaccine design which takes advantage of the wealth of genomic, proteomic, and transcriptomic datasets, using biological samples from humans or animals with immunity to the disease of interest and functionally relevant screening criteria to identify the key antigens and their epitopes targeted by protective immune responses. With this focus, we can improve on nature. In the case of malaria, validated human challenge models provide a valuable resource for genome-based antigen discovery. Moreover, this information can be integrated with other omic-based and cutting-edge immunological based approaches. For example, systems immunology could be applied to identify immune signatures that distinguish a protective immune response from a non-protective one. The many technological and intellectual advances in the omics era offer great potential for the development of rationally designed vaccines against malaria as well as other pathogens that have thus far proved elusive.

\section{REFERENCES}

Acharya, P., Pallavi, R., Chandran, S., Chakravarti, H., Middha, S., Acharya, J., et al. (2009). A glimpse into the clinical proteome of human malaria parasites Plasmodium falciparum and Plasmodium vivax. Proteom. Clin. Appl. 3, 13141325. doi: 10.1002/prca.200900090

Akram, A., and Inman, R. D. (2012). Immunodominance: a pivotal principle in host response to viral infections. Clin. Immunol. 143, 99-115. doi: 10.1016/j.clim.2012.01.015

Anders, R. F. (2011). The case for a subunit vaccine against malaria. Trends Parasitol. 27, 330-334. doi: 10.1016/j.pt.2011.04.003

Annoura, T., Ploemen, I. H., van Schaijk, B. C., Sajid, M., Vos, M. W., van Gemert, G. J., et al. (2012). Assessing the adequacy of attenuation of genetically modified malaria parasite vaccine candidates. Vaccine 30, 2662-2670. doi: 10.1016/j.vaccine.2012.02.010

Baird, J. K. (1998). Age-dependent characteristics of protection versus susceptibility to Plasmodium falciparum. Ann. Trop. Med. Parasitol. 92, 367-390. doi: 10.1080/00034989859366

Ballou, W. R., and Cahill, C. P. (2007). Two decades of commitment to malaria vaccine development: GlaxoSmithKline biologicals. Am. J. Trop. Med. Hyg. 77, 289-295.

Barry, A. E., and Arnott, A. (2014). Strategies for designing and monitoring malaria vaccines targeting diverse antigens. Front. Immunol. 5:359. doi: 10.3389/fimmu.2014.00359

Bartfai, R., Hoeijmakers, W. A., Salcedo-Amaya, A. M., Smits, A. H., Janssen-Megens, E., Kaan, A., et al. (2010). H2A.Z demarcates intergenic regions of the Plasmodium falciparum epigenome that are dynamically marked by $\mathrm{H} 3 \mathrm{~K} 9 \mathrm{ac}$ and $\mathrm{H} 3 \mathrm{~K} 4 \mathrm{me} 3$. PLoS Pathog. 6:e1001223. doi: 10.1371/journal.ppat.1001223

Baxby, D. (1999). Edward Jenner's Inquiry; a bicentenary analysis. Vaccine 17, 301307. doi: 10.1016/S0264-410X(98)00207-2

Beaudoin, R. L., Strome, C. P. A., Mitchell, F., and Tubergen, T. A. (1977). Plasmodium berghei: immunization of mice against the ANKA strain using the unaltered sporozoite as an antigen. Exp. Parasitol. 42, 1-5. doi: 10.1016/00144894(77)90054-6

Bedoui, S., Whitney, P. G., Waithman, J., Eidsmo, L., Wakim, L., Caminschi, I., et al. (2009). Cross-presentation of viral and self antigens by skin-derived CD103+ dendritic cells. Nat. Immunol. 10, 488-495. doi: 10.1038/ni.1724

Behet, M. C., Foquet, L., van Gemert, G. J., Bijker, E. M., Meuleman, P., LerouxRoels, G., et al. (2014). Sporozoite immunization of human volunteers under chemoprophylaxis induces functional antibodies against pre-erythrocytic stages of Plasmodium falciparum. Malar. J. 13, 136. doi: 10.1186/1475-2875-13-136

Belnoue, E., Costa, F. T., Frankenberg, T., Vigario, A. M., Voza, T., Leroy, N., et al. (2004). Protective T cell immunity against malaria liver stage after vaccination with live sporozoites under chloroquine treatment. J. Immunol. 172, 2487-2495. doi: 10.4049/jimmunol.172.4.2487

Bergmann-Leitner, E. S., Mease, R. M., De La Vega, P., Savranskaya, T., Polhemus, M., Ockenhouse, C., et al. (2010). Immunization with pre-erythrocytic antigen CelTOS from Plasmodium falciparum elicits cross-species protection against heterologous challenge with Plasmodium berghei. PLoS ONE 5:e12294. doi: 10.1371/journal.pone.0012294

Bijker, E. M., Bastiaens, G. J., Teirlinck, A. C., van Gemert, G. J., Graumans, W., van de Vegte-Bolmer, M., et al. (2013). Protection against malaria after immunization by chloroquine prophylaxis and sporozoites is mediated by preerythrocytic immunity. Proc. Natl. Acad. Sci. U.S.A. 110, 7862-7867. doi: 10.1073/pnas.1220360110

Bijker, E. M., Teirlinck, A. C., Schats, R., van Gemert, G. J., van de Vegte-Bolmer, M., van Lieshout, L., et al. (2014). Cytotoxic markers associate with protection 
against malaria in human volunteers immunized with Plasmodium falciparum sporozoites. J. Infect. Dis. doi: 10.1093/infdis/jiu293 [Epub ahead of print].

Birkett, A. J., Moorthy, V. S., Loucq, C., Chitnis, C. E., and Kaslow, D. C. (2013). Malaria vaccine R\&D in the decade of vaccines: breakthroughs, challenges and opportunities. Vaccine 31(Suppl. 2), B233-B243. doi: 10.1016/j.vaccine.2013.02.040

Bozdech, Z., Llinas, M., Pulliam, B. L., Wong, E. D., Zhu, J., and DeRisi, J. L. (2003). The transcriptome of the intraerythrocytic developmental cycle of Plasmodium falciparum. PLoS Biol. 1:E5. doi: 10.1371/journal.pbio.0000005

Bozdech, Z., Mok, S., Hu, G., Imwong, M., Jaidee, A., Russell, B., et al. (2008) The transcriptome of Plasmodium vivax reveals divergence and diversity of transcriptional regulation in malaria parasites. Proc. Natl. Acad. Sci. U.S.A. 105, 16290-16295. doi: 10.1073/pnas.0807404105

Brooks, A., Briet, O. J., Hardy, D., Steketee, R., and Smith, T. A. (2012). Simulated impact of RTS,S/AS01 vaccination programs in the context of changing malaria transmission. PLoS ONE 7:e32587. doi: 10.1371/journal.pone.0032587

Bruder, J. T., Angov, E., Limbach, K. J., and Richie, T. L. (2010). Molecular vaccines for malaria. Hum. Vaccin. 6, 54-77. doi: 10.4161/hv.6.1.10463

Butler, N. S., Schmidt, N. W., Vaughan, A. M., Aly, A. S., Kappe, S. H., and Harty, J. T. (2011). Superior antimalarial immunity after vaccination with late liver stage-arresting genetically attenuated parasites. Cell Host Microbe 9, 451-462. doi: 10.1016/j.chom.2011.05.008

Carlton, J. M., Adams, J. H., Silva, J. C., Bidwell, S. L., Lorenzi, H., Caler, E., et al. (2008). Comparative genomics of the neglected human malaria parasite Plasmodium vivax. Nature 455, 757-763. doi: 10.1038/nature07327

Carlton, J. M., Angiuoli, S. V., Suh, B. B., Kooij, T. W., Pertea, M., Silva, J. C., et al (2002). Genome sequence and comparative analysis of the model rodent malaria parasite Plasmodium yoelii yoelii. Nature 419, 512-519. doi: 10.1038/nature01099

Casares, S., and Richie, T. L. (2009). Immune evasion by malaria parasites: a challenge for vaccine development. Curr. Opin. Immunol. 21, 321-330. doi: 10.1016/j.coi.2009.05.015

Chen, L., Keitany, G. J., Peng, X., Gibson, C., Mohar, I., Vignali, M., et al. (2014) Identification of pre-erythrocytic malaria antigens that target hepatocytes for killing in vivo and contribute to protection elicited by whole-parasite vaccination. PLOS ONE 9:e102225. doi: 10.1371/journal.pone.0102225

Cherif, M. S., Shuaibu, M. N., Kodama, Y., Kurosaki, T., Helegbe, G. K., Kikuchi, M. et al. (2014). Nanoparticle formulation enhanced protective immunity provoked by PYGPI8p-transamidase related protein (PyTAM) DNA vaccine in Plasmodium yoelii malaria model. Vaccine 32, 1998-2006. doi: 10.1016/j.vaccine.2014.01.005

Cohen, S., McGregor, I., and Carrington, S. (1961). Gamma-globulin and acquired immunity to human malaria. Nature 192, 733-737. doi: 10.1038/192733a0

Crompton, P. D., Kayala, M. A., Traore, B., Kayentao, K., Ongoiba, A., Weiss, G. E., et al. (2010a). A prospective analysis of the Ab response to Plasmodium falciparum before and after a malaria season by protein microarray. Proc. Natl. Acad. Sci. U.S.A. 107, 6958-6963. doi: 10.1073/pnas.1001323107

Crompton, P. D., Pierce, S. K., and Miller, L. H. (2010b). Advances and challenges in malaria vaccine development. J. Clin. Invest. 120, 4168-4178. doi $10.1172 / \mathrm{JCI} 44423$

Daily, J. P., Scanfeld, D., Pochet, N., Le Roch, K., Plouffe, D., Kamal, M., et al. (2007) Distinct physiological states of Plasmodium falciparum in malaria-infected patients. Nature 450, 1091-1095. doi: 10.1038/nature06311

Doolan, D. L. (2011). Plasmodium immunomics. Int. J. Parasitol. 41, 3-20. doi: 10.1016/j.ijpara.2010.08.002

Doolan, D. L., Apte, S. H., and Proietti, C. (2014). Genome-based vaccine design: the promise for malaria and other infectious diseases. Int. J. Parasitol. 44, 901-913. doi: 10.1016/j.ijpara.2014.07.010

Doolan, D. L., Dobano, C., and Baird, J. K. (2009). Acquired immunity to malaria. Clin. Microbiol. Rev. 22, 13-36. doi: 10.1128/CMR.00025-08

Doolan, D. L., and Hoffman, S. L. (1997). Multi-gene vaccination against malaria: a multistage, multi-immune response approach. Parasitol. Today 13, 171-178. doi: 10.1016/S0169-4758(97)01040-5

Doolan, D. L., and Hoffman, S. L. (2000). The complexity of protective immunity against liver-stage malaria. J. Immunol. 165, 1453-1462. doi: 10.4049/jimmunol.165.3.1453

Doolan, D. L., Hoffman, S. L., Southwood, S., Wentworth, P. A., Sidney, J., Chesnut, R. W., et al. (1997). Degenerate cytotoxic T cell epitopes from P. falciparum restricted by multiple $H L A-A$ and $H L A-B$ supertype alleles. Immunity 7, 97-112. doi: 10.1016/S1074-7613(00)80513-0
Doolan, D. L., Mu, Y., Unal, B., Sundaresh, S., Hirst, S., Valdez, C., et al. (2008) Profiling humoral immune responses to $P$. falciparum infection with protein microarrays. Proteomics 8, 4680-4694. doi: 10.1002/pmic.200800194

Doolan, D. L., Southwood, S., Chesnut, R., Appella, E., Gomez, E., Richards, A., et al. (2000). HLA-DR-promiscuous T cell epitopes from Plasmodium falciparum preerythrocytic-stage antigens restricted by multiple HLA class II alleles. J. Immunol. 165, 1123-1137. doi: 10.4049/jimmunol.165.2.1123

Doolan, D. L., Southwood, S., Freilich, D. A., Sidney, J., Graber, N. L., Shatney, L., et al. (2003). Identification of Plasmodium falciparum antigens by antigenic analysis of genomic and proteomic data. Proc. Natl. Acad. Sci. U.S.A. 100, 99529957. doi: 10.1073/pnas.1633254100

Duffy, P. E., Sahu, T., Akue, A., Milman, N., and Anderson, C. (2012). Pre-erythrocytic malaria vaccines: identifying the targets. Expert Rev. Vaccines 11, 1261-1280. doi: 10.1586/erv.12.92

Duncan, C. J., and Hill, A. V. (2011). What is the efficacy of the RTS, S malaria vaccine? BMJ 343, d7728. doi: 10.1136/bmj.d7728

Elliott, S. R., Kuns, R. D., and Good, M. F. (2005). Heterologous immunity in the absence of variant-specific antibodies after exposure to subpatent infection with blood-stage malaria. Infect. Immun. 73, 2478-2485. doi: 10.1128/IAI.73.4.24782485.2005

Epstein, J. E., Tewari, K., Lyke, K. E., Sim, B. K., Billingsley, P. F., Laurens, M. B., et al. (2011). Live attenuated malaria vaccine designed to protect through hepatic CD8(+) T cell immunity. Science 334, 475-480. doi: 10.1126/science.1211548

Florens, L., Liu, X., Wang, Y., Yang, S., Schwartz, O., Peglar, M., et al. (2004). Proteomics approach reveals novel proteins on the surface of malaria-infected erythrocytes. Mol. Biochem. Parasitol. 135, 1-11. doi: 10.1016/j.molbiopara.2003.12.007

Florens, L., Washburn, M. P., Raine, J. D., Anthony, R. M., Grainger, M., Haynes, J. D., et al. (2002). A proteomic view of the Plasmodium falciparum life cycle. Nature 419, 520-526. doi: 10.1038/nature01107

Florent, I., Charneau, S., and Grellier, P. (2004). Plasmodium falciparum genes differentially expressed during merozoite morphogenesis. Mol. Biochem. Parasitol. 135, 143-148. doi: 10.1016/j.molbiopara.2003.12.010

Florent, I., Porcel, B. M., Guillaume, E., Da Silva, C., Artiguenave, F., Marechal, E., et al. (2009). A Plasmodium falciparum FcB1-schizont-EST collection providing clues to schizont specific gene structure and polymorphism. BMC Genom. 10:235. doi: 10.1186/1471-2164-10-235

Friedrich, T. C., Valentine, L. E., Yant, L. J., Rakasz, E. G., Piaskowski, S. M., Furlott, J. R., et al. (2007). Subdominant CD8 + T-cell responses are involved in durable control of AIDS virus replication. J. Virol. 81, 3465-3476. doi: 10.1128/JVI.023 92-06

Friesen, J., Silvie, O., Putrianti, E. D., Hafalla, J. C., Matuschewski, K., and Borrmann, S. (2010). Natural immunization against malaria: causal prophylaxis with antibiotics. Sci. Transl. Med. 2, 40ra49. doi: 10.1126/scitranslmed.3001058

Gardner, M. J., Hall, N., Fung, E., White, O., Berriman, M., Hyman, R. W., et al. (2002). Genome sequence of the human malaria parasite Plasmodium falciparum. Nature 419, 498-511. doi: 10.1038/nature01097

Good, M. F., and Doolan, D. L. (2010). Malaria vaccine design: immunological considerations. Immunity 33, 555-566. doi: 10.1016/j.immuni.2010.10.005

Good, M. F., Kumar, S., and Miller, L. H. (1988). The real difficulties for malaria sporozoite vaccine development: nonresponsiveness and antigenic variation. Immunol. Today 9, 351-355. doi: 10.1016/0167-5699(88)91 336-9

Good, M. F., Reiman, J. M., Rodriguez, I. B., Ito, K., Yanow, S. K., El-Deeb, I. M., et al. (2013). Cross-species malaria immunity induced by chemically attenuated parasites. J. Clin. Invest. 123, 3353-3362. doi: 10.1172/JCI66634

Gruner, A. C., Mauduit, M., Tewari, R., Romero, J. F., Depinay, N., Kayibanda, M., et al. (2007). Sterile protection against malaria is independent of immune responses to the circumsporozoite protein. PLoS ONE 2:e1371. doi: 10.1371/journal.pone.0001371

Gunasekera, A. M., Patankar, S., Schug, J., Eisen, G., Kissinger, J., Roos, D., etal. (2004). Widespread distribution of antisense transcripts in the Plasmodium falciparum genome. Mol. Biochem. Parasitol. 136, 35-42. doi: 10.1016/j.molbiopara.2004.02.007

Gunasekera, A. M., Patankar, S., Schug, J., Eisen, G., and Wirth, D. F. (2003). Drug-induced alterations in gene expression of the asexual blood forms of Plasmodium falciparum. Mol. Microbiol. 50, 1229-1239. doi: 10.1046/j.13652958.2003.03787.x 
Gwadz, R. W., Cochrane, A. H., Nussenzweig, V., and Nussenzweig, R. S. (1979). Preliminary studies on vaccination of rhesus monkeys with irradiated sporozoites of Plasmodium knowlesi and characterization of surface antigens of these parasites. Bull. World Health Organ. 57(Suppl. 1), 165-173.

Hafalla, J. C., Bauza, K., Friesen, J., Gonzalez-Aseguinolaza, G., Hill, A. V., and Matuschewski, K. (2013). Identification of targets of CD8(+) T cell responses to malaria liver stages by genome-wide epitope profiling. PLoS Pathog. 9:e1003303. doi: 10.1371/journal.ppat.1003303

Hall, B. F., and Fauci, A. S. (2009). Malaria control, elimination, and eradication: the role of the evolving biomedical research agenda. J. Infect. Dis. 200, 1639-1643. doi: $10.1086 / 646611$

Hall, N., Karras, M., Raine, J. D., Carlton, J. M., Kooij, T. W., Berriman, M., etal. (2005). A comprehensive survey of the Plasmodium life cycle by genomic, transcriptomic, and proteomic analyses. Science 307, 82-86. doi: $10.1126 /$ science. 1103717

Hirunpetcharat, C., and Good, M. F. (1998). Deletion of Plasmodium bergheispecific CD4+ $\mathrm{T}$ cells adoptively transferred into recipient mice after challenge with homologous parasite. Proc. Natl. Acad. Sci. U.S.A. 95, 1715-1720. doi: 10.1073/pnas.95.4.1715

Hoffman, S. L., Goh, L. M., Luke, T. C., Schneider, I., Le, T. P., Doolan, D. L., et al (2002). Protection of humans against malaria by immunization with radiationattenuated Plasmodium falciparum sporozoites. J. Infect. Dis. 185, 1155-1164. doi: $10.1086 / 339409$

Jenner, E. (1798). An Inquiry into Causes and Effects of Variolae Vaccinae, a Disease, Discovered in some of the Western Countries of England, Particularly Gloucestershire, and know by the name of Cow Pox. London: Printed by Sampson Low.

Kariu, T., Ishino, T., Yano, K., Chinzei, Y., and Yuda, M. (2006). CelTOS, a novel malarial protein that mediates transmission to mosquito and vertebrate hosts. Mol. Microbiol. 59, 1369-1379. doi: 10.1111/j.1365-2958.2005.05024.x

Khan, S. M., Franke-Fayard, B., Mair, G. R., Lasonder, E., Janse, C. J., Mann, M., et al. (2005). Proteome analysis of separated male and female gametocytes reveals novel sex-specific Plasmodium biology. Cell 121, 675-687. doi 10.1016/j.cell.2005.03.027

Kirkman, L. A., and Deitsch, K. W. (2012). Antigenic variation and the generation of diversity in malaria parasites. Curr. Opin. Microbiol. 15, 456-462. doi 10.1016/j.mib.2012.03.003

Kloverpris, H., Karlsson, I., Bonde, J., Thorn, M., Vinner, L., Pedersen, A. E., et al (2009). Induction of novel CD8+ T-cell responses during chronic untreated HIV1 infection by immunization with subdominant cytotoxic T-lymphocyte epitopes. AIDS 23, 1329-1340. doi: 10.1097/QAD.0b013e32832d9b00

Klysik, J. (2001). Concept of immunomics: a new frontier in the battle for gene function? Acta Biotheor. 49, 191-202. doi: 10.1023/A:1011901410166

Kumar, K. A., Sano, G., Boscardin, S., Nussenzweig, R. S., Nussenzweig, M. C., Zavala, F., etal. (2006). The circumsporozoite protein is an immunodominant protective antigen in irradiated sporozoites. Nature 444, 937-940. doi: 10.1038 /nature05361

Labaied, M., Harupa, A., Dumpit, R. F., Coppens, I., Mikolajczak, S. A., and Kappe, S. H. (2007). Plasmodium yoelii sporozoites with simultaneous deletion of P52 and P36 are completely attenuated and confer sterile immunity against infection. Infect. Immun. 75, 3758-3768. doi: 10.1128/IAI.00225-07

Langhorne, J., Ndungu, F. M., Sponaas, A. M., and Marsh, K. (2008). Immunity to malaria: more questions than answers. Nat. Immunol. 9, 725-732. doi: 10.1038/ni.f.205

Lasonder, E., Ishihama, Y., Andersen, J. S., Vermunt, A. M., Pain, A., Sauerwein, R. W., et al. (2002). Analysis of the Plasmodium falciparum proteome by high-accuracy mass spectrometry. Nature 419, 537-542. doi: 10.1038/nature01111

Lasonder, E., Janse, C. J., van Gemert, G. J., Mair, G. R., Vermunt, A. M., Douradinha, B. G., et al. (2008). Proteomic profiling of Plasmodium sporozoite maturation identifies new proteins essential for parasite development and infectivity. PLoS Pathog. 4:e1000195. doi: 10.1371/journal.ppat.1000195

Le Roch, K. G., Johnson, J. R., Florens, L., Zhou, Y., Santrosyan, A., Grainger, M., et al. (2004). Global analysis of transcript and protein levels across the Plasmodium falciparum life cycle. Genome Res. 14, 2308-2318. doi: 10.1101/gr.2523904

Le Roch, K. G., Zhou, Y., Blair, P. L., Grainger, M., Moch, J. K., Haynes, J. D., et al. (2003). Discovery of gene function by expression profiling of the malaria parasite life cycle. Science 301, 1503-1508. doi: 10.1126/science.1087025

Limbach, K., Aguiar, J., Gowda, K., Patterson, N., Abot, E., Sedegah, M., et al. (2011). Identification of two new protective pre-erythrocytic malaria vaccine antigen candidates. Malar. J. 10, 65. doi: 10.1186/1475-2875-10-65
Lindner, S. E., Swearingen, K. E., Harupa, A., Vaughan, A. M., Sinnis, P., Moritz, R. L., et al. (2013). Total and putative surface proteomics of malaria parasite salivary gland sporozoites. Mol. Cell. Proteom. 12, 1127-1143. doi: 10.1074/mcp.M112.024505

Liu, W., Li, Y., Learn, G. H., Rudicell, R. S., Robertson, J. D., Keele, B. F., et al. (2010). Origin of the human malaria parasite Plasmodium falciparum in gorillas. Nature 467, 420-425. doi: 10.1038/nature09442

Llinas, M., Bozdech, Z., Wong, E. D., Adai, A. T., and DeRisi, J. L. (2006). Comparative whole genome transcriptome analysis of three Plasmodium falciparum strains. Nucleic Acids Res. 34, 1166-1173. doi: 10.1093/nar/gkj517

Lopez-Barragan, M. J., Lemieux, J., Quinones, M., Williamson, K. C., Molina-Cruz, A., Cui, K., et al. (2011). Directional gene expression and antisense transcripts in sexual and asexual stages of Plasmodium falciparum. BMC Genom. 12:587. doi: 10.1186/1471-2164-12-587

Mackinnon, M. J., and Marsh, K. (2010). The selection landscape of malaria parasites. Science 328, 866-871. doi: 10.1126/science.1185410

Matuschewski, K., Hafalla, J. C., Borrmann, S., and Friesen, J. (2011). Arrested Plasmodium liver stages as experimental anti-malaria vaccines. Hum. Vaccin. 7(Suppl.), 16-21. doi: 10.4161/hv.7.0.14557

Mauduit, M., Tewari, R., Depinay, N., Kayibanda, M., Lallemand, E., Chavatte, J. M., et al. (2010). Minimal role for the circumsporozoite protein in the induction of sterile immunity by vaccination with live rodent malaria sporozoites. Infect. Immun. 78, 2182-2188. doi: 10.1128/IAI.01415-09

Menard, R. (2005). Medicine: knockout malaria vaccine? Nature 433, 113-114. doi: $10.1038 / 433113 a$

Mikolajczak, S. A., Lakshmanan, V., Fishbaugher, M., Camargo, N., Harupa, A., Kaushansky, A., et al. (2014). A next-generation genetically attenuated Plasmodium falciparum parasite created by triple gene deletion. Mol. Ther. 22, 1707-1715. doi: $10.1038 / \mathrm{mt} .2014 .85$

Mikolajczak, S. A., Silva-Rivera, H., Peng, X., Tarun, A. S., Camargo, N., Jacobs-Lorena, V., et al. (2008). Distinct malaria parasite sporozoites reveal transcriptional changes that cause differential tissue infection competence in the mosquito vector and mammalian host. Mol. Cell. Biol. 28, 6196-6207. doi: 10.1128/MCB.00553-08

Mishra, S., Rai, U., Shiratsuchi, T., Li, X., Vanloubbeeck, Y., Cohen, J., et al. (2011). Identification of non-CSP antigens bearing CD8 epitopes in mice immunized with irradiated sporozoites. Vaccine 29, 7335-7342. doi: 10.1016/j.vaccine.2011.07.081

Moorthy, V. S., and Kieny, M. P. (2010). Reducing empiricism in malaria vaccine design. Lancet Infect. Dis. 10, 204-211. doi: 10.1016/S1473-3099(09)70329-9

Mueller, A. K., Labaied, M., Kappe, S. H., and Matuschewski, K. (2005). Genetically modified Plasmodium parasites as a protective experimental malaria vaccine. Nature 433, 164-167. doi: 10.1038/nature03188

Nahrendorf, W., Scholzen, A., Bijker, E. M., Teirlinck, A. C., Bastiaens, G. J., Schats, R., et al. (2014). Memory B-cell and antibody responses induced by Plasmodium falciparum sporozoite immunization. J. Infect. Dis. doi: 10.1093/infdis/jiu354 [Epub ahead of print]

Nganou-Makamdop, K., and Sauerwein, R. W. (2013). Liver or blood-stage arrest during malaria sporozoite immunization: the later the better? Trends Parasitol. 29, 304-310. doi: 10.1016/j.pt.2013.03.008

Nunes, J. K., Cardenas, V., Loucq, C., Maire, N., Smith, T., Shaffer, C., et al. (2013). Modeling the public health impact of malaria vaccines for developers and policymakers. BMC Infect. Dis. 13:295. doi: 10.1186/1471-2334-13-295

Nussenzweig, R. S., Vanderberg, J., Most, H., and Orton, C. (1967). Protective immunity produced by the injection of X-irradiated sporozoites of Plasmodium berghei. Nature 216, 160-162. doi: 10.1038/216160a0

Oehring, S. C., Woodcroft, B. J., Moes, S., Wetzel, J., Dietz, O., Pulfer, A., et al. (2012). Organellar proteomics reveals hundreds of novel nuclear proteins in the malaria parasite Plasmodium falciparum. Genome Biol. 13, R108. doi: 10.1186/gb-201213-11-r108

Okell, L. C., Ghani, A. C., Lyons, E., and Drakeley, C. J. (2009). Submicroscopic infection in Plasmodium falciparum-endemic populations: a systematic review and meta-analysis. J. Infect. Dis. 200, 1509-1517. doi: 10.1086/644781

Olotu, A., Fegan, G., Wambua, J., Nyangweso, G., Awuondo, K. O., Leach, A., et al. (2013). Four-year efficacy of RTS,S/AS01E and its interaction with malaria exposure. N. Engl. J. Med. 368, 1111-1120. doi: 10.1056/NEJMoa12 07564

Orjih, A. U., Cochrane, A. H., and Nussenzweig, R. S. (1982). Comparative studies on the immunogenicity of infective and attenuated sporozoites 
of Plasmodium berghei. Trans. R. Soc. Trop. Med. Hyg. 76, 57-61. doi: 10.1016/0035-9203(82)90019-0

Osier, F. H., Fegan, G., Polley, S. D., Murungi, L., Verra, F., Tetteh, K. K., et al. (2008). Breadth and magnitude of antibody responses to multiple Plasmodium falciparum merozoite antigens are associated with protection from clinical malaria. Infect. Immun. 76, 2240-2248. doi: 10.1128/IAI.01585-07

Otto, T. D., Wilinski, D., Assefa, S., Keane, T. M., Sarry, L. R., Bohme, U., et al. (2010) New insights into the blood-stage transcriptome of Plasmodium falciparum using RNA-Seq. Mol. Microbiol. 76, 12-24. doi: 10.1111/j.1365-2958.2009.07026.x

Owusu-Agyei, S., Koram, K. A., Baird, J. K., Utz, G. C., Binka, F. N., Nkrumah, F. K., et al. (2001). Incidence of symptomatic and asymptomatic Plasmodium falciparum infection following curative therapy in adult residents of northern Ghana. Am. J. Trop. Med. Hyg. 65, 197-203.

Pain, A., Bohme, U., Berry, A. E., Mungall, K., Finn, R. D., Jackson, A. P., et al (2008). The genome of the simian and human malaria parasite Plasmodium knowlesi. Nature 455, 799-803. doi: 10.1038/nature07306

Patankar, S., Munasinghe, A., Shoaibi, A., Cummings, L. M., and Wirth, D. F. (2001). Serial analysis of gene expression in Plasmodium falciparum reveals the global expression profile of erythrocytic stages and the presence of antisense transcripts in the malarial parasite. Mol. Biol. Cell 12, 3114-3125. doi: 10.1091/mbc.12.10.3114

Pierce, S. K., and Miller, L. H. (2009). World Malaria Day 2009: what malaria knows about the immune system that immunologists still do not. J. Immunol. 182, 5171-5177. doi: 10.4049/jimmunol.0804153

Plowe, C. V., Alonso, P., and Hoffman, S. L. (2009). The potential role of vaccines in the elimination of falciparum malaria and the eventual eradication of malaria. J. Infect. Dis. 200, 1646-1649. doi: 10.1086/646613

Pombo, D. J., Lawrence, G., Hirunpetcharat, C., Rzepczyk, C., Bryden, M., Cloonan, N., et al. (2002). Immunity to malaria after administration of ultra-low doses of red cells infected with Plasmodium falciparum. Lancet 360, 610-617. doi: 10.1016/S0140-6736(02)09784-2

Purcell, L. A., Wong, K. A., Yanow, S. K., Lee, M., Spithill, T. W., and Rodriguez, A. (2008a). Chemically attenuated Plasmodium sporozoites induce specific immune responses, sterile immunity and cross-protection against heterologous challenge. Vaccine 26, 4880-4884. doi: 10.1016/j.vaccine.2008.07.017

Purcell, L. A., Yanow, S. K., Lee, M., Spithill, T. W., and Rodriguez, A. (2008b). Chemical attenuation of Plasmodium berghei sporozoites induces sterile immunity in mice. Infect. Immun. 76, 1193-1199. doi: 10.1128/IAI.01399-07

Putrianti, E. D., Silvie, O., Kordes, M., Borrmann, S., and Matuschewski, K. (2009). Vaccine-like immunity against malaria by repeated causal-prophylactic treatment of liver-stage Plasmodium parasites. J. Infect. Dis. 199, 899-903. doi: $10.1086 / 597121$

Roberts, L., and Enserink, M. (2007). Malaria. Did they really say... eradication? Science 318, 1544-1545. doi: 10.1126/science.318.5856.1544

Roestenberg, M., McCall, M., Hopman, J., Wiersma, J., Luty, A. J., van Gemert, G. J. et al. (2009). Protection against a malaria challenge by sporozoite inoculation. $N$ Engl. J. Med. 361, 468-477. doi: 10.1056/NEJMoa0805832

Roestenberg, M., Teirlinck, A. C., McCall, M. B., Teelen, K., Makamdop, K. N., Wiersma, J., et al. (2011). Long-term protection against malaria after experimental sporozoite inoculation: an open-label follow-up study. Lancet 377, 1770-1776 doi: 10.1016/S0140-6736(11)60360-7

Roobsoong, W., Roytrakul, S., Sattabongkot, J., Li, J., Udomsangpetch, R., and Cui, L. (2011). Determination of the Plasmodium vivax schizont stage proteome. J. Proteom. 74, 1701-1710. doi: 10.1016/j.jprot.2011.03.035

Rovira-Graells, N., Gupta, A. P., Planet, E., Crowley, V. M., Mok, S., Ribas de Pouplana, L., etal. (2012). Transcriptional variation in the malaria parasite Plasmodium falciparum. Genome Res. 22, 925-938. doi: 10.1101/gr.1296 92.111

Ruckwardt, T. J., Luongo, C., Malloy, A. M., Liu, J., Chen, M., Collins, P. L., et al. (2010). Responses against a subdominant CD8+ T cell epitope protect against immunopathology caused by a dominant epitope. J. Immunol. 185, 4673-4680. doi: 10.4049/jimmunol.1001606

Rueckert, C., and Guzman, C. A. (2012). Vaccines: from empirical development to rational design. PLoS Pathog. 8:e1003001. doi: 10.1371/journal.ppat.1003001

Sabchareon, A., Burnouf, T., Ouattara, D., Attanath, P., Bouharoun-Tayoun, H., Chantavanich, P., et al. (1991). Parasitologic and clinical human response to immunoglobulin administration in Falciparum malaria. Am. J. Trop. Med. Hyg. $45,297-308$
Sacci, J. B. Jr., Ribeiro, J. M., Huang, F., Alam, U., Russell, J. A., Blair, P. L., et al. (2005) Transcriptional analysis of in vivo Plasmodium yoelii liver stage gene expression. Mol. Biochem. Parasitol. 142, 177-183. doi: 10.1016/j.molbiopara.2005. 03.018

Sam-Yellowe, T. Y., Florens, L., Wang, T., Raine, J. D., Carucci, D. J., Sinden, R., et al. (2004). Proteome analysis of rhoptry-enriched fractions isolated from Plasmodium merozoites. J. Proteome Res. 3, 995-1001. doi: 10.1021/ pr049926m

Schmidt, N. W., Butler, N. S., Badovinac, V. P., and Harty, J. T. (2010). Extreme CD8 $\mathrm{T}$ cell requirements for anti-malarial liver-stage immunity following immunization with radiation attenuated sporozoites. PLoS Pathog. 6:e1000998. doi: 10.1371/journal.ppat.1000998

Schmidt, N. W., Podyminogin, R. L., Butler, N. S., Badovinac, V. P., Tucker, B. J., Bahjat, K. S., et al. (2008). Memory CD8 T cell responses exceeding a large but definable threshold provide long-term immunity to malaria. Proc. Natl. Acad. Sci. U.S.A. 105, 14017-14022. doi: 10.1073/pnas.0805452105

Schofield, L., Villaquiran, J., Ferreira, A., Schellekens, H., Nussenzweig, R., and Nussenzweig, V. (1987). Gamma interferon, CD8+ T cells and antibodies required for immunity to malaria sporozoites. Nature 330, 664-666. doi: 10.1038/330664a0 Schussek, S., Trieu, A., and Doolan, D. L. (2014). Genome- and proteome-wide screening strategies for antigen discovery and immunogen design. Biotechnol. Adv. 32, 403-414. doi: 10.1016/j.biotechadv.2013.12.006

Schwartz, L., Brown, G. V., Genton, B., and Moorthy, V. S. (2012). A review of malaria vaccine clinical projects based on the WHO rainbow table. Malar. J. 11, 11. doi: 10.1186/1475-2875-11-11

Seder, R. A., Chang, L. J., Enama, M. E., Zephir, K. L., Sarwar, U. N., Gordon, I. J., et al. (2013). Protection against malaria by intravenous immunization with a nonreplicating sporozoite vaccine. Science 341, 1359-1365. doi: $10.1126 /$ science. 1241800

Sercarz, E. E., Lehmann, P. V., Ametani, A., Benichou, G., Miller, A., and Moudgil, K. (1993). Dominance and crypticity of T cell antigenic determinants. Annu. Rev. Immunol. 11, 729-766. doi: 10.1146/annurev.iy.11.040193.003501

Sherman, I. W. (2009). The Elusive Malaria Vaccine: Miracle or Mirage? Washington, DC: American Society for Microbiology.

Siau, A., Silvie, O., Franetich, J. F., Yalaoui, S., Marinach, C., Hannoun, L., et al. (2008). Temperature shift and host cell contact up-regulate sporozoite expression of Plasmodium falciparum genes involved in hepatocyte infection. PLoS Pathog. 4:e1000121. doi: 10.1371/journal.ppat.1000121

Silvestrini, F., Lasonder, E., Olivieri, A., Camarda, G., van Schaijk, B., Sanchez, M., et al. (2010). Protein export marks the early phase of gametocytogenesis of the human malaria parasite Plasmodium falciparum. Mol. Cell. Proteom. 9, 1437-1448. doi: 10.1074/mcp.M900479-MCP200

Spring, M., Murphy, J., Nielsen, R., Dowler, M., Bennett, J. W., Zarling, S., et al. (2013). First-in-human evaluation of genetically attenuated Plasmodium falciparum sporozoites administered by bite of Anopheles mosquitoes to adult volunteers. Vaccine 31, 4975-4983. doi: 10.1016/j.vaccine.2013.08.007

Stephens, R., Albano, F. R., Quin, S., Pascal, B. J., Harrison, V., Stockinger, B., et al. (2005). Malaria-specific transgenic CD4+ T cells protect immunodeficient mice from lethal infection and demonstrate requirement for a protective threshold of antibody production for parasite clearance. Blood 106, 1676-1684. doi: 10.1182/blood-2004-10-4047

Tachibana, S., Sullivan, S. A., Kawai, S., Nakamura, S., Kim, H. R., Goto, N. et al. (2012). Plasmodium cynomolgi genome sequences provide insight into Plasmodium vivax and the monkey malaria clade. Nat. Genet. 44, 1051-1055. doi: 10.1038/ng.2375

Takala, S. L., and Plowe, C. V. (2009). Genetic diversity and malaria vaccine design, testing and efficacy: preventing and overcoming 'vaccine resistant malaria.' Parasite Immunol. 31, 560-573. doi: 10.1111/j.1365-3024.2009.01138.x

Tarun, A. S., Peng, X., Dumpit, R. F., Ogata, Y., Silva-Rivera, H., Camargo, N., et al. (2008). A combined transcriptome and proteome survey of malaria parasite liver stages. Proc. Natl. Acad. Sci. U.S.A. 105, 305-310. doi: 10.1073/pnas.0710780104

Taylor, S. M., Cerami, C., and Fairhurst, R. M. (2013). Hemoglobinopathies: slicing the Gordian knot of Plasmodium falciparum malaria pathogenesis. PLoS Pathog. 9:e1003327. doi: 10.1371/journal.ppat.1003327

Tran, T. M., Li, S., Doumbo, S., Doumtabe, D., Huang, C. Y., Dia, S., et al. (2013). An intensive longitudinal cohort study of Malian children and adults reveals no evidence of acquired immunity to Plasmodium falciparum infection. Clin. Infect Dis. 57, 40-47. doi: 10.1093/cid/cit174 
Trieu, A., Kayala, M. A., Burk, C., Molina, D. M., Freilich, D. A., Richie, T. L., et al. (2011). Sterile protective immunity to malaria is associated with a panel of novel P. falciparum antigens. Mol. Cell. Proteomics 10, M111 007948. doi: 10.1074/mcp.M111.007948

Tsuji, M. (2010). A retrospective evaluation of the role of $\mathrm{T}$ cells in the development of malaria vaccine. Exp. Parasitol. 126, 421-425. doi: 10.1016/j.exppara.2009.11.009

Tuells, J. (2012). Vaccinology: the name, the concept, the adjectives. Vaccine 30, 5491-5495. doi: 10.1016/j.vaccine.2012.06.059

Vignali, M., Armour, C. D., Chen, J., Morrison, R., Castle, J. C., Biery, M. C., et al. (2011). NSR-seq transcriptional profiling enables identification of a gene signature of Plasmodium falciparum parasites infecting children. J. Clin. Invest. 121, 1119-1129. doi: 10.1172/JCI43457

Wang, C. W., Mwakalinga, S. B., Sutherland, C. J., Schwank, S., Sharp, S., Hermsen C. C., et al. (2010). Identification of a major rif transcript common to gametocytes and sporozoites of Plasmodium falciparum. Malar. J. 9, 147. doi: 10.1186/14752875-9-147

Watanabe, J., Wakaguri, H., Sasaki, M., Suzuki, Y., and Sugano, S. (2007). Comparasite: a database for comparative study of transcriptomes of parasites defined by full-length cDNAs. Nucleic Acids Res. 35, D431-D438. doi: 10.1093/nar/gkl1039

Weiss, W. R., and Jiang, C. G. (2012). Protective CD8+ T lymphocytes in primates immunized with malaria sporozoites. PLOS ONE 7:e31247. doi: 10.1371/journal.pone.0031247

Westenberger, S. J., McClean, C. M., Chattopadhyay, R., Dharia, N. V., Carlton, J. M., Barnwell, J. W., et al. (2010). A systems-based analysis of Plasmodium vivax lifecycle transcription from human to mosquito. PLoS Negl. Trop. Dis. 4:e653. doi: 10.1371/journal.pntd.0000653

Winzeler, E. A. (2006). Applied systems biology and malaria. Nat. Rev. Microbiol. 4, 145-151. doi: 10.1038/nrmicro1327

World Health Organization [WHO]. (2006). State of the Art of New Vaccine Research and Development. Geneva: World Health Organization.

World Health Organization [WHO]. (2012). Global Vaccine Action Plan 2011 -2020. Geneva: WHO Press.

World Health Organization [WHO]. (2014a). The 10 Leading Causes of Death in the World, 2000 and 2012. Geneva: World Health Organization.
World Health Organization [WHO]. (2014b). Malaria Vaccine Rainbow Tables. Geneva: World Health Organization.

Xu, H., Wipasa, J., Yan, H., Zeng, M., Makobongo, M. O., Finkelman, F. D., et al. (2002). The mechanism and significance of deletion of parasite-specific CD4(+) T cells in malaria infection. J. Exp. Med. 195, 881-892. doi: 10.1084/jem.20011174 Young, J. A., Fivelman, Q. L., Blair, P. L., de la Vega, P., Le Roch, K. G., Zhou, Y., et al. (2005). The Plasmodium falciparum sexual development transcriptome: a microarray analysis using ontology-based pattern identification. Mol. Biochem. Parasitol. 143, 67-79. doi: 10.1016/j.molbiopara.2005.05.007

Zepp, F. (2010). Principles of vaccine design - lessons from nature. Vaccine 28(Suppl. 3), C14-C24. doi: 10.1016/j.vaccine.2010.07.020

Zhang, X., Tolzmann, C. A., Melcher, M., Haas, B. J., Gardner, M. J., Smith, J. D., et al. (2011). Branch point identification and sequence requirements for intron splicing in Plasmodium falciparum. Eukaryot. Cell 10, 1422-1428. doi: 10.1128/EC. 05193-11

Zhang, Y., Qi, Y., Li, J., Liu, S., Hong, L., Lin, T., et al. (2012). A new malaria antigen produces partial protection against Plasmodium yoelii challenge. Parasitol. Res. 110, 1337-1345. doi: 10.1007/s00436-011-2630-y

Conflict of Interest Statement: The authors declare that the research was conducted in the absence of any commercial or financial relationships that could be construed as a potential conflict of interest.

Received: 02 October 2014; accepted: 06 December 2014; published online: 22 January 2015.

Citation: Proietti C and Doolan DL (2015) The case for a rational genome-based vaccine against malaria. Front. Microbiol. 5:741. doi: 10.3389/fmicb.2014.00741

This article was submitted to Microbial Immunology, a section of the journal Frontiers in Microbiology.

Copyright $\odot 2015$ Proietti and Doolan. This is an open-access article distributed under the terms of the Creative Commons Attribution License (CC BY). The use, distribution or reproduction in other forums is permitted, provided the original author(s) or licensor are credited and that the original publication in this journal is cited, in accordance with accepted academic practice. No use, distribution or reproduction is permitted which does not comply with these terms. 